\title{
Mitochondrial Quality Control and Disease: Insights into Ischemia-Reperfusion Injury
}

\author{
Anthony R. Anzell ${ }^{1,2,3} \cdot$ Rita Maizy $^{1}$ - Karin Przyklenk ${ }^{1,2,3} \cdot$ Thomas H. Sanderson $^{1,2,3}$
}

Received: 31 January 2017 / Accepted: 20 March 2017 /Published online: 11 April 2017

(C) The Author(s) 2017. This article is published with open access at Springerlink.com

\begin{abstract}
Mitochondria are key regulators of cell fate during disease. They control cell survival via the production of ATP that fuels cellular processes and, conversely, cell death via the induction of apoptosis through release of pro-apoptotic factors such as cytochrome $\mathrm{C}$. Therefore, it is essential to have stringent quality control mechanisms to ensure a healthy mitochondrial network. Quality control mechanisms are largely regulated by mitochondrial dynamics and mitophagy. The processes of mitochondrial fission (division) and fusion allow for damaged mitochondria to be segregated and facilitate the equilibration of mitochondrial components such as DNA, proteins, and metabolites. The process of mitophagy are responsible for the degradation and recycling of damaged mitochondria. These mitochondrial quality control mechanisms have been well studied in chronic and acute pathologies such as Parkinson's disease, Alzheimer's disease, stroke, and acute myocardial infarction, but less is known about how these two processes interact and contribute to specific pathophysiologic states. To date, evidence for the role of mitochondrial quality control in acute and chronic disease is divergent and suggests that mitochondrial quality control processes can serve both survival and death functions depending on the disease state. This review aims to provide a synopsis of the molecular mechanisms involved in mitochondrial quality control,
\end{abstract}

Thomas H. Sanderson

tsanders@med.wayne.edu

1 Department of Emergency Medicine, Wayne State University School of Medicine, Detroit, MI 48201, USA

2 Cardiovascular Research Institute, Wayne State University School of Medicine, Detroit, MI 48201, USA

3 Department of Physiology, Wayne State University School of Medicine, Detroit, MI 48201, USA to summarize our current understanding of the complex role that mitochondrial quality control plays in the progression of acute vs chronic diseases and, finally, to speculate on the possibility that targeted manipulation of mitochondrial quality control mechanisms may be exploited for the rationale design of novel therapeutic interventions.

Keywords Brain $\cdot$ Mitophagy $\cdot$ Ischemia $\cdot$ Reperfusion · Mitochondria $\cdot$ Mitochondrial dynamics

\section{Introduction: Mitophagic Balance in Acute and Chronic Disease}

Cardiovascular and neurologic diseases are leading causes of morbidity and mortality in the USA [1]. Cardiovascular disease can result in acute injuries sustained by both the heart and the brain in the form of acute myocardial infarction (AMI) and stroke. AMI and stroke are induced by a cessation of blood flow (ischemia), caused by blockage of one or more of the coronary or cerebral arteries that supply the heart or brain. This cessation of blood flow will subsequently lead to tissue hypoxia or anoxia and, ultimately, necrotic cell death (characterized by cellular swelling and membrane rupture due to energy failure). It is well known that although restoration of blood flow (reperfusion) is essential to salvage ischemic tissue, this can also, paradoxically, exacerbate damage from several cellular alterations including excessive reactive oxygen species (ROS) production from mitochondria [2-4]. ROS production will lead to mitochondrial damage and, ultimately, mitochondrial failure and predominantly apoptosis (typically occurring via the intrinsic or mitochondrial pathway) $[5,6]$. Ultimately, cell death observed during ischemia and reperfusion in both heart and brain occurs over a broad spectrum of cell death phenotypes depending on the duration and severity 
of the ischemic insult. This process occurring at the level of the tissue has been termed lethal ischemia/reperfusion (I/R) injury.

In addition to acute injuries such as stroke or AMI, the incidence of chronic neuropathologies (including, for example, Parkinson's disease (PD) and Alzheimer's disease (AD)) are on the rise as the average lifespan continues to increase $[7,8]$. PD and $\mathrm{AD}$ are neurodegenerative diseases that affect different parts of the brain and are typically seen in the elderly, although inherited mutations may also lead to disease in younger patients. PD is a movement disorder, and while many forms of hereditary and acquired PD have unique mechanistic causes, all result in a pathologic dysfunction and death of dopaminergic neurons. $\mathrm{AD}$ is a neurodegenerative disorder that affects the elderly and causes memory loss and declined cognitive function. The pathological factors of the disease consist of the presence of amyloid- $\beta$ plaques and neurofibrillary tangles in the brain [9]. Although I/R injury, Parkinson's disease, and Alzheimer's disease differ in terms of their etiology, the literature suggests that loss of mitochondrial integrity plays a central role in each of these disease processes.

It is well established that mitochondria are key regulators of cell fate, controlling survival (via the production of ATP that fuels cellular processes) and, conversely, death (via the induction of apoptosis). Indeed, mitochondrial dysfunction has been well characterized as a precursor to cell death [10-13]. Therefore, it is essential to have stringent control mechanisms regulating the quality of mitochondria to avoid the pathologic effects of dysfunctional mitochondria on the cell.

While the negative roles of mitochondrial failure and apoptosis are well documented, much less is known of the causal role of mitochondrial quality control in disease and the potentially nuanced role for these mechanisms in different disease settings. Importantly, evidence suggests a divergent role of mitochondrial quality control in acute vs. chronic disease. Defects in the mechanisms that regulate quality of mitochondria are recognized to play a large role in chronic diseases such as PD and AD, but, to date, their role in the acute setting of I/R injury is poorly understood. In contrast, evidence supports a salutary role for mitochondrial quality control in acute cardiac and neurologic injury, suggesting that quality control mechanisms can serve both survival and death functions depending on the nature of the disease. This review aims to (i) discuss the molecular mechanisms involved in mitochondrial quality control including mitochondrial dynamics and mitophagy (Fig. 1), (ii) detail the role that mitochondrial quality control plays in chronic and acute neurodegenerative and cardiovascular diseases, and (iii) provide a better understanding of the intricacies and balance of this process in the progression of acute vs chronic diseases.

\section{The Axes of Mitochondrial Quality Control}

\section{Mitochondrial Dynamics: to Divide or Not to Divide?}

Within the cell, mitochondria exist in an ever-changing dynamic state, where mitochondrial networks are constantly elongating and dividing (i.e., mitochondrial fusion and fission, respectively). The balance of these two events provides an equilibrium of small fragmented mitochondria and long interconnected mitochondrial networks and is thought to be essential for mitochondrial homeostasis, cell stability, and cell survival (Fig. 1) [14, 15]. Fission plays a role in segregating dysfunctional mitochondria that contain damaged proteins, destabilized membranes, and mutated or damaged mitochondrial DNA (mtDNA) [16-20]. Fusion, in contrast, has been shown to aid in equilibration of matrix metabolites, intact mtDNA, and even membrane components such as complex I of the electron transport chain [16, 21-24]. Fission and fusion are both regulated by a family of dynamin-related proteins (DRPs). These proteins are unique in that they are large selfassembling GTPases that also possess the capability of assembly-stimulated GTP hydrolysis [25]. Through the work of DRPs, the mitochondrial network can be in constant communication to ensure a healthy connected network, while at the same time allowing the distribution of mitochondria to specific sites of the cell via transport on actin or microtubule networks [26, 27].

\section{Mitochondrial Fission}

The master mediator of fission is dynamin-related protein 1 (Drp1), which has been shown to be essential for noncytokinetic mitochondrial division [16, 28]. Drp1 is distributed diffusely throughout the cytosol and, when activated through posttranslational modifications (predominantly phosphorylation/dephosphorylation), translocates to the outer mitochondrial membrane via actin and microtubule mechanisms [29-33]. These post-translational modifications, described in detail below, include phosphorylation/dephosphorylation, ubiquitination, and sumoylation, in a cell-specific manner [34, 35]. Once positioned on the outer mitochondrial membrane, Drp1 interacts with four mitochondrial-bound proteins that serve as Drp1 receptors (mitochondrial dynamic proteins of 49 and $51 \mathrm{kDa}$ (Mid49 and Mid51), mitochondrial fission protein 1 (Fis1), and mitochondrial fission factor (Mff), where it constricts and cleaves the mitochondria (Fig. 2a) [36-38]. Fis1 is an $18-\mathrm{kDa}$ adaptor protein anchored to the outer mitochondrial membrane and has been implicated in recruiting Drp1, as well as modulating the assembly of the fission complex [39, 40]. Fis1 is thought to be required for mitochondrial fission, although this remains controversial as other groups have found it to be dispensable in the fission process $[38,41,42]$.

Fission is regulated by numerous post-translational modifications of Drp1 as well as endoplasmic reticulum (ER)-mitochondrial contact sites. Phosphorylation/dephosphorylation 


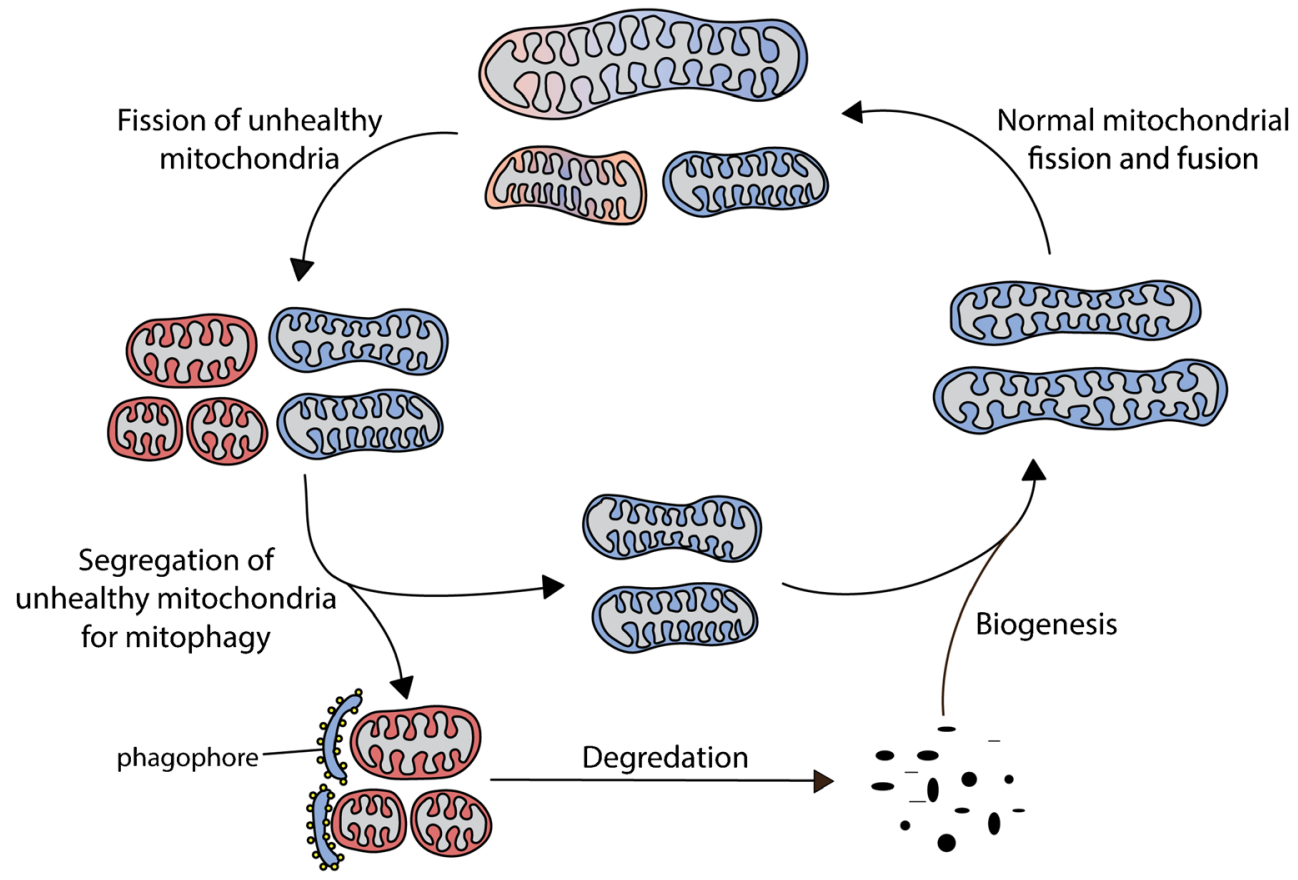

Fig. 1 The mitochondrial quality control cycle. The mitochondrial quality control cycle involves a dynamic process of fission, fusion, mitophagy, and biogenesis. When mitochondria become depolarized or dysfunctional, they are marked for degradation. Once marked, the unhealthy component of the mitochondria will undergo fission from the healthy mitochondrial network. Certain damaged mitochondria can fuse with other healthy mitochondria in an attempt to salvage that mitochondrion, but typically, dysfunctional mitochondria will undergo mitophagy. When the dysfunctional mitochondria are segregated from the healthy mitochondrial network, mitochondria will accumulate mitophagy markers that will recruit the phagophore. The phagophore will attach to the dysfunctional mitochondria, mature into an autophagosome, fuse with the lysosome to form the autolysosome, and degrade the mitochondria. Once degraded, the cell will recycle the amino acids and fatty acids to enable the remaining healthy mitochondrial network to grow and divide through biogenesis is one of the main regulators of Drp1 and is carried out at two different serine sites located 20 amino acids apart (Ser616 and Ser637) [43]. Phosphorylation of Ser616 is associated with Drp1 activation and is phosphorylated by cyclin-dependent kinase 1 (CDK1), extracellular signal-regulated kinase (ERK1/2), and protein kinase C delta (PKCס) [44-46]. CDK1 induces mitochondrial fragmentation during mitosis [44]. ERK1/2 and PKC $\delta$ induces Drp1-mediated mitochondrial fission via increases in ROS production during hyperglycemic conditions and hypertensive neuroencephalopathy, respectively $[45,46]$. Ser637 is phosphorylated by protein kinase A (PKA), calcium/calmodulin-dependent protein kinase 1 alpha (CAMKI $\alpha$ ), and the Rho-associated coil-containing protein kinase 1 (ROCK1) [34, 35, 47]. PKA-phosphorylated Drp1 has been shown to have decreased GTPase activity and result in decreased fission during starvation, stress, or exercise. Studies in neurons and cardiac tissue exposed to oxygenglucose deprivation (OGD) and ischemia-reperfusion, respectively, demonstrate calcineurin-mediated dephosphorylation of Ser637, subsequently leading to Drp1 activation, mitochondrial fission, and apoptosis [33, 48, 49]. Conversely, CAMKI $\alpha$ phosphorylation of Ser637 results in enhanced fission during conditions of high extracellular $\mathrm{K}^{+}$(inducing $\mathrm{Ca}^{2+}$ influx) in primary rat hippocampal neurons [33, 35, 47]. Additionally, ROCK1 phosphorylation of Ser637 has been demonstrated to induce mitochondrial fission in podocytes and endothelial cells of mice with metabolic syndrome and diabetes [34]. Phosphorylation of the same residue leading to opposite effects points to the complexity of Drp1 regulation that is likely dependent on cell type, extracellular conditions, as well as intracellular status.

Recent literature also suggests a role for the ER in mitochondrial fission. Studies conducted in both yeast and mammalian cells have shown that ER tubules will wrap around the mitochondria and mediate constriction before Drp1 recruitment via the ER-localized inverted formin 2 (INF2) mechanisms [50, 51]. INF2 is thought to drive initial mitochondrial constriction that provides sites for subsequent Drp1 recruitment and secondary constriction [51]. The multiple mechanisms involved in the regulation of fission underscore the complexity of this process and may provide insight into potential mechanisms by which dysregulated mitochondrial dynamics may interact with disease processes.

\section{Mitochondrial Fusion}

Fusion is mediated by three different GTPases: optic atrophy 1 (Opa1), mitofusin 1 (Mfn1), and mitofusin 2 (Mfn2) [24]. Both $\mathrm{Mfn} 1$ and Mfn2 mediate fusion of the outer mitochondrial membranes, while Opal mediates the fusion of the inner 


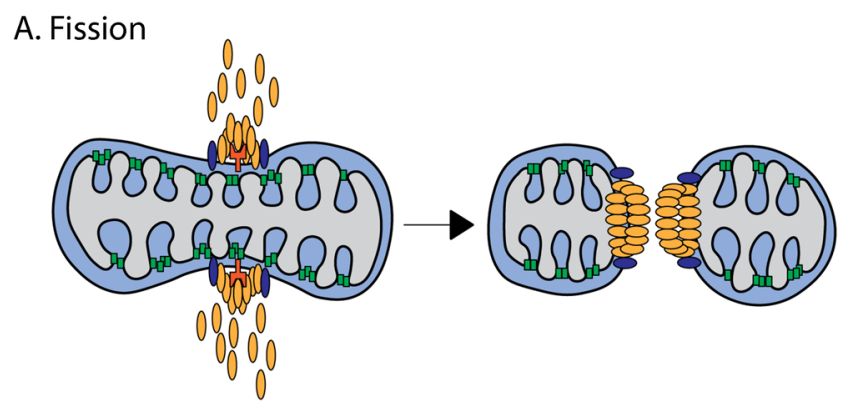

B. Fusion

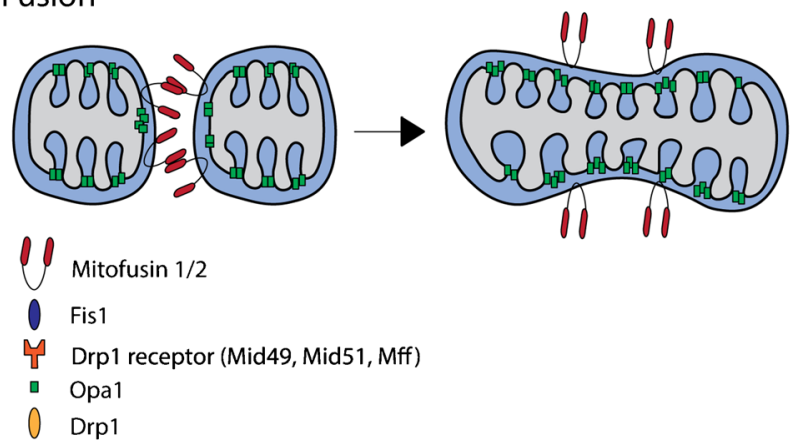

Fig. 2 Mitochondrial dynamics. a Fission is mediated by a family of dynamin-related proteins (Drp). Activated Drp1 translocates from the cytosol to the mitochondrial membrane where it interacts with Drp1 receptors (Mid 49, Mid51, Mff) and Fis1 to create the fission complex. Drp1 oligomers constrict and divide the mitochondria. b Fusion is mediated through the mitofusins (Mfn1/2) and optic atrophy 1 (Opa1). The mitofusins mediate the fusion of the outer mitochondrial membrane, while Opal is thought to mediate fusion of the inner mitochondrial membrane. Mitofusins are anchored to the outer mitochondrial membrane and interact with each other and form a hemifusion stalk. The stalk then grows into a lipidic hole and finally reestablishes membrane continuity. Opa1 forms a fusion pore for the inner mitochondrial membrane via its cardiolipin binding domain

mitochondrial membrane, along with its role in maintaining normal inner membrane cristae structure [52-54]. Mitofusins, which are required for fusion, are anchored to the outer mitochondrial membrane where they interact and form a hemifusion stalk to initiate the joining of two mitochondrial membranes [16, 55]. The stalk then grows and creates a lipidic hole as well as a hemifusion diaphragm to reestablish membrane continuity. Finally, a fusion pore is made for inner membrane fusion via the lipid binding domain in Opa1 that is specific for cardiolipin $[55,56]$ (Fig. 2b).

Fusion of the inner and outer mitochondrial membrane is mediated mainly through proteolytic cleavage and ubiquitination, respectively. Opal, in mammals, consists of eight different isoforms generated by alternative splicing of three of the 30 Opal exons [57, 58]. Membrane-bound long (L)-Opal can be further processed via two proteolytic cleavage sites (S1 and S2), generating short (S)-Opal forms [54]. Proteolytic processing is carried out predominantly through two intermembrane space AAA proteases (ATPases associated with diverse cellular activities): (i) overlapping with m-AAA (OMA1) cleaving at the S1 site and (ii) yeast mitochondrial DNA escape 1-like (YME1L) cleaving at the S2 site [59, 60]. Every (L)-Opalisoform contains a S1 cleavage site, while about half of the (L)-Opa1 isoforms contain both S1 and S2 cleavage sites [61, 62]. Under normal physiological conditions, $\mathrm{S} 1$ and $\mathrm{S} 2$ are constitutively cleaved to produce a 50/50 ratio of (L)-Opa1 and (S)-Opa1. The balance in Opal isoforms is thought to mediate the balance between mitochondrial fission and fusion [63]. Under pathophysiologic conditions, such as membrane depolarization, low levels of ATP, or dysfunctional quality control mechanisms, the balance is tipped and the remaining (L)-Opa1 are cleaved by Oma1 resulting in mitochondrial fragmentation [64-68]. Mitofusins are regulated mainly by ubiquitin-mediated degradation, specifically through the PTEN-induced kinase (PINK1) and Parkin-mediated ubiquitination pathway during mitophagy [69]. This pathway will be discussed later in further detail. Abnormalities in proteolytic cleavage of Opal or ubiquitination of the mitofusins result in impaired fusion, changes in cristae architecture, and favor a fragmented mitochondrial phenotype.

\section{Mitophagy: Out with the Old, In with the New}

Autophagy is the catabolic process of cellular components including cytosolic protein aggregates and organelles such as mitochondria that are sequestered in a double-membrane structure called an autophagosome [70, 71]. There are three distinct subtypes of autophagy [72]. Macroautophagy (typically referred to as autophagy) is the process of taking damaged proteins and organelles from the cytoplasm to the lysosome for degradation via an intermediate vesicle termed the autophagosome (summarized in Fig. 3). Macroautophagy typically involves the degradation of large cellular components such as organelles through both selective and nonselective mechanisms. In contrast, in so-called microautophagy, particles are directly taken up by the lysosome (no intermediate vesicle) by direct engulfment where they are degraded. Lastly, chaperone-mediated autophagy involves targeting dysfunctional proteins to be taken across the lysosomal membrane with the aid of the cytosolic chaperone heat shock cognate $70 \mathrm{kDa}$ protein (HSC-70). The protein-chaperone protein complex then interacts with a specific lysosomal membrane receptor, lysosomal-associated membrane protein $2 \mathrm{~A}$ (LAMP-2A), resulting in their degradation [73, 74].

The process of mitochondrial degradation through macroautophagy has been termed mitophagy. Mitophagy occurs through several different pathways (summarized in Fig. 4) that all involve (i) detection of dysfunctional mitochondria, (ii) segregation from the healthy mitochondrial network, (iii) recruitment of the phagophore, and (iv) degradation through autophagic processes. Mitophagy, in concert with mitochondrial biogenesis, ensures a healthy mitochondrial network through mitochondrial turnover. Clearance of dysfunctional mitochondria is critical to limit cellular damage via ROS production and subsequent apoptosis. Mitophagic proteins, specifically Parkin, are critical for eliminating mitochondria with deleterious mtDNA mutations via 


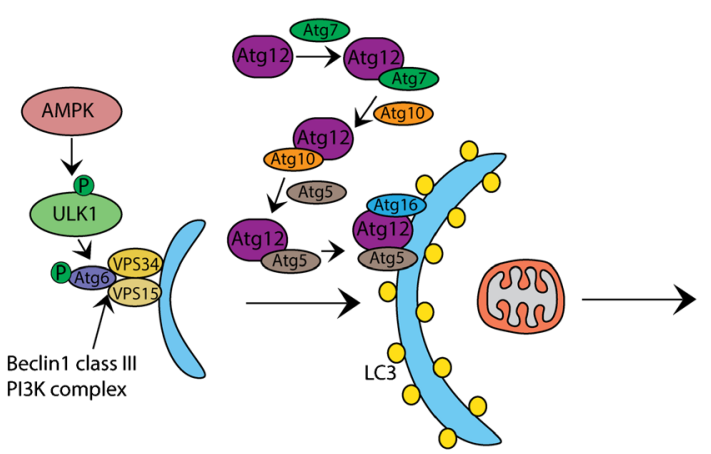

Nucleation

Elongation
Fig. 3 Phases of Autophagy. Autophagy is carried out in four different phases: nucleation, elongation, sequestration, and degradation. Nucleation of the isolation membrane is initiated by the phosphorylation of the ULk1 complex by AMPK. ULK1 will then recruit several autophagosome-related proteins for nucleation of the phagophore via phosphorylation of beclin 1 in the PI3K complex. Elongation or the extension of the autophagosome membrane is mediated by two ubiquitin-like systems involving the transferring of Atg12 from $\operatorname{Atg} 7$ to $\operatorname{Atg} 10$ and finally Atg5 where it forms a dimeric complex with

mitophagy [20]. This suggests that mitophagy is selective and plays pivotal role in the maintaining a functional population of mitochondria.

\section{Autophagy: the Four Phases}

There are four phases in the development of an autophagosome: (i) nucleation, (ii) elongation, (iii) sequestration and maturation, followed by (iv) fusion and degradation (Fig. 3) [75, 76].

Nucleation of the isolation membrane is initiated first through the phosphorylation of the Unc-51 like autophagy activating kinase 1 (ULK1) complex, typically by 5' AMPactivated protein kinase (AMPK) [77]. Once phosphorylated, the ULK1 complex will recruit several different autophagosome-related proteins (Atg) to the autophagosome formation site. In addition, ULK1 also phosphorylates beclin 1 (Atg6) which, in turn, initiates the activity of the class III phosphotidylinositol 3-kinase (PI3K) complex (beclin 1 and vacuolar proteins sorting 34 and 15 (VPS34 and VPS15)) for nucleation of the phagophore [77-79].

Elongation of the phagophore is mediated by two ubiquitin-like systems (ULS) [77]. In the Atg5-Atg12 conjugation system (first of the two ULS), Atg12 is activated by Atg7 (an E1-like enzyme) and is then transferred to Atg10 (an E2-like enzyme) that is on the target protein Atg5. This Atg5Atg12 will form a dimeric complex with Atg16, which will target the phagophore membrane. The second ULS involves the cleavage of Atg8 by Atg4 (cysteine protease) and, subsequently, the processing by the ubiquitin-like enzymes Atg7 and Atg3 [77]. Together, these ULS, along with light chain 3 (LC3), extend the autophagosome membrane.

Sequestration is the process by which the isolation membrane encircles the damaged organelle. This is mediated through the binding of LC3 to a variety of different proteins/ receptors that detect damaged organelles [78, 80, 81]. LC3 is processed by a cysteine protease to its cytosolic form, LC3I. Cytosolic LC 3 I then conjugates with phosphotidylethanolamine (PE) associated with the inner and outer membrane of the phagophore to form LC3II [82]. The phagophore will then continue to elongate until it completely engulfs its cargo and matures into an autophagosome.

In the degradation phase, the autophagosome then fuses with the lysosome, resulting in the degradation of its cargo via acid hydrolase enzymes $[75,76]$. In the fusion process, soluble NSF attachment protein receptor (SNARE) proteins, endosomal coating proteins (COPs), the endosomal sorting complex require for transport (ESCRT III) complex, the homotypic fusion and protein sorting (HOPS) complex, LAMP proteins, GTPase Rab proteins, the beclin 1 binding protein Rubicon, and chaperon HSP70 family proteins have all been implicated to play contributing roles [83-90]. More specifically, Chen et al. reported that tectonin beta-propeller repeat-containing protein 1 (TECPR1) of the lysosome binds phosphotidylinositol 3-phosphate upon conjugation of Atg12Atg5 to promote autophagosome-lysosome fusion [91]. After degradation, the cargo's amino acids and lipids can then be reused for synthesis of new organelles.

\section{Identification of Dysfunctional Mitochondria: the Pathways} of Mitophagy

Four pathways have been identified that detect dysfunctional mitochondria and recruit autophagosomes for degradation (summarized in Fig. 4). The most well-known pathway is PINK1/Parkin-mediated mitophagy [92, 93], 
A. PINK1/Parkin

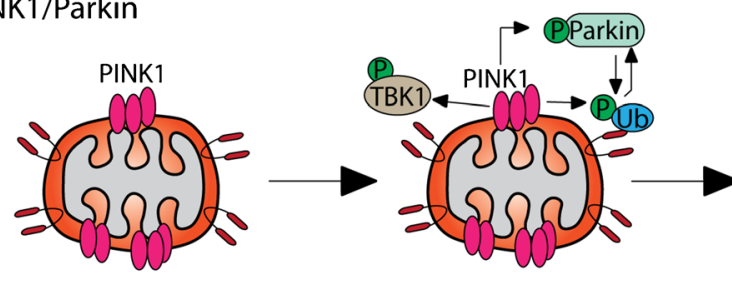

B. BNIP3/NIX
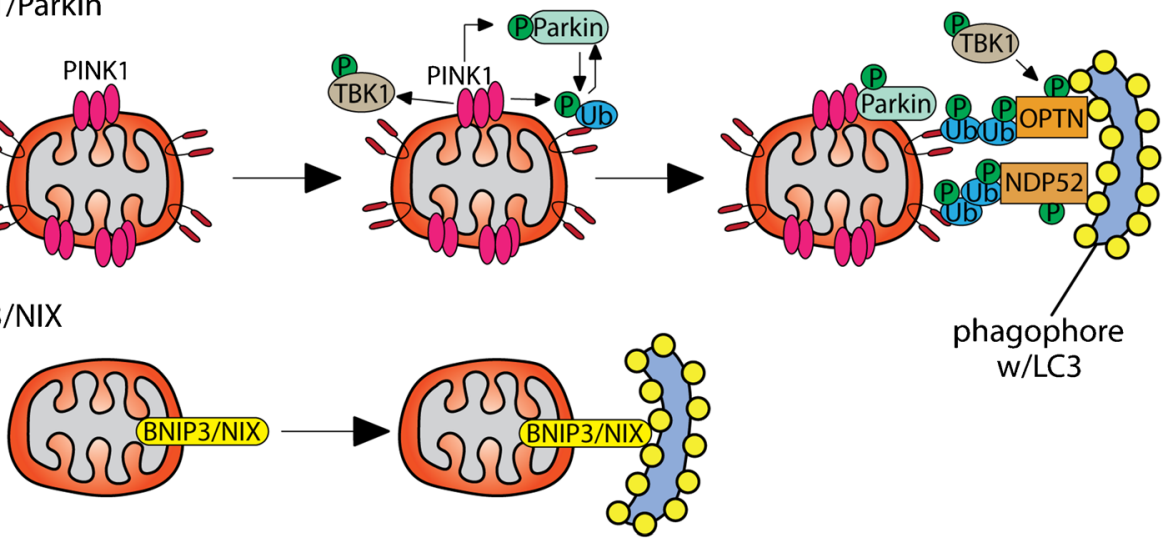

C. FUNDC1

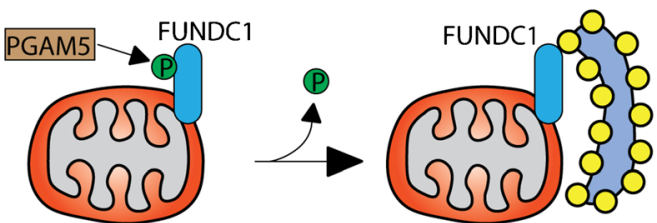

D. Cardiolipin
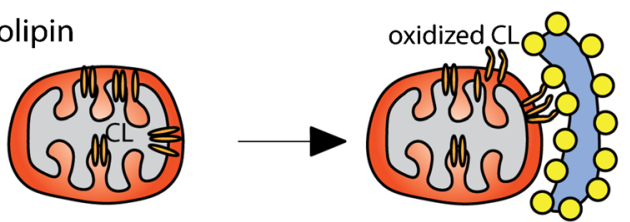

Fig. 4 Mitophagic pathways. a In dysfunctional mitochondria, PINK1 accumulates in the outer mitochondrial membrane. Accumulations of PINK1 induce a concerted signaling cascade involving the simultaneous recruitment and phosphorylation of the E3 ubiquitin ligase Parkin, ubiquitin, and TBK1. Phosphorylation by PINK1 as well as phospho-Ser65-ubiquitin activates Parkin and leads to ubiquitination of outer mitochondrial membrane proteins in a feed forward process. Phagophore recruitment and binding are then mediated by OPTN and NDP52 with its ubiquitin and LC3 binding domains. b BNIP3/Nix are

named for its role in the pathogenesis of Parkinson's disease. PINK1 contains a mitochondrial targeting domain such that, in healthy mitochondria, it is (i) transported into the intermembrane space (IMS) through the translocase of the outer mitochondrial membrane (TOM), then (ii) integrated into the inner mitochondrial membrane via insertion into the translocase of the inner mitochondrial membrane (TIM) [94], and (iii) rapidly processed and degraded by the mitochondrial membrane peptidase and presinilin-associated rhomboid-like protease (PARL). Under healthy conditions, this rapid degradation serves to keep the mitochondrial concentration of PINK1 low [95]. However, TIM-mediated import of protein relies on a steady mitochondrial membrane potential. When mitochondria are depolarized, PINK1 can no longer be inserted into the mitochondria, inhibiting its proteolytic cleavage and subsequent degradation [96]. PINK1 then accumulates on depolarized mitochondria, where it localized on the outer mitochondrial membrane and serve as mitophagy receptors and bind directly to the phagosome via LC3. c FUNDC1 localizes on the outer mitochondrial membrane and acts as a receptor for mitophagy under hypoxic condition. During hypoxia, PGAM5 dephosphorylates FUNDC1 and activates mitophagy via LC3 binding on the phagophore. $\mathbf{d}$ Cardiolipin localizes mainly in the inner leaflet of the inner mitochondrial membrane, specifically around the folds of the cristae. When cardiolipin is oxidized, it is externalized to the outer mitochondrial membrane where it is recognized by LC3 of the phagophore

phosphorylates and activates a myriad of proteins including Parkin, ubiquitin, and TANK binding kinase 1 (TBK1) [92, 97-101] (Fig. 4a).

Parkin is an E3 ubiquitin ligase that is activated by phosphorylation by PINK at Ser65 within its ubiquitin like domain $[102,103]$. In addition, Parkin has also been reported to be activated by PINK1-dependent phosphorylation of ubiquitin at Ser65 [99, 101, 104]. When activated, Parkin will ubiquitinate numerous outer mitochondrial membrane proteins including mitofusins and voltage-dependent anion channel (VDAC) [69, 105, 106]. Interestingly, the ubiquitin chains generated by Parkin are major targets of PINK1 phosphorylation, allowing Parkin retention on mitochondria, providing a feed forward mechanism to promote mitophagy [98, 104]. Concurrently, PINK1 also phosphorylates TBK1 at Ser172, promoting the phosphorylation of three different autophagy adaptor proteins: p62 (also known as sequestrome 1 (SQSTM1)), optineurin (OPTN), and nuclear dot protein 
(NDP52) [107-109]. The aggregation of dysfunctional mitochondria is mediated via p62, while OPTN and NDP52 serve as receptors for the phagophore via ubiquitin and LC3 binding domains [108-113] (Fig. 4a). Histone deacetylase 6 (HDAC6) will also translocate upon ubiquitination of outer mitochondrial membrane proteins and has been shown to enhance fusion of the autophagosome and lysosome $[114,115]$.

Mitophagy can also be regulated in a receptor-mediated fashion. One of these pathways is through BNIP3/NIX, which are B cell CLL/lymphoma 2 (BCL-2)-related proteins. These proteins play a dual role by both (i) inducing mitochondrial apoptosis and (ii) localizing to the mitochondrial membrane and acting as autophagy receptors where they can directly bind to LC3 [116-120] (Fig. 4b). This pathway is distinct from the PINK1/Parkin-mediated mitophagy in that PINK1/ Parkin requires depolarized mitochondria to initiate mitophagy, whereas BNIP3 can activate mitophagy in mitochondria that have a stable membrane potential $[92,93,121]$. BNIP3 does recruit Parkin to mitochondria, and it has been shown that Parkin-deficient myocytes display a reduction in mitophagy despite overexpression of BNIP3 [122]. Although these proteins have dual function in activating mitophagy or inducing apoptosis, it is unclear how they are recruited for each divergent role.

FUN14 domain containing 1 (FUNDC1) is an outer mitochondrial membrane protein that mediates mitophagy through receptor binding with $\mathrm{LC} 3$ and has been implicated to play a role in hypoxia-mediated mitophagy [123] (Fig. 4c). FUNDC1 is regulated by casein kinase 2 (CK2) and the mitochondrial serine/threonine protein phosphatase PGAM5 [124]: Specifically, CK2 phosphorylates FUNDC1 to inhibit its function, while during hypoxia, PGAM5 phosphatase dephosphorylates FUNDC1 to activate its binding to LC3 and thus promote mitophagy. This pathway has been shown to be related to both PINK1/Parkin and BNIP3 primarily through PGAM5. PGAM5 phosphatase activity is required for PINK1 stabilization as well as PINK1/Parkin-mediated mitophagy, and PGAM5-deficient mice develop Parkinson's disease [125]. BNIP3 has also been shown to be activated in hypoxia and induce mitophagy [126]. Whether or not these three pathways communicate with one another is still a question that needs further investigation.

Cardiolipin is a lipid predominantly localized to the inner mitochondrial membrane, is involved in mitochondrial metabolism, and interestingly, has also recently been implicated in receptor-mediated mitophagy $[127,128]$. When oxidized, cardiolipin undergoes redistribution and externalization to the surface of damaged mitochondria where it is recognized by LC3 [128] (Fig. 4d). Nucleoside diphosphate kinase-D (NDPK-D (NM23-H4)), a hexameric intermembrane space protein, mediates the externalization of cardiolipin in artificially depolarized mitochondria [129]. How this process may interact with PINK1/Parkin pathway is still unknown but may provide novel insight to a potential role for cardiolipin signaling in pathologies involving mitochondrial membrane depolarization, i.e., I/R injury.

\section{Mitochondrial Biogenesis}

Mitochondrial biogenesis refers to the growth and division of pre-existing mitochondria. After mitochondria are degraded, the existing mitochondrial pool needs to continue growing to keep pace with energy demands of the cell. The increase in mitochondrial content involves an array of processes that include protein and lipid synthesis driven by both nuclear and mtDNA transcription. The double-stranded circular mtDNA is about $16.5 \mathrm{~kb}$ in length and contains 37 genes that encode for 13 proteins (subunits of electron transport chain complexes), 22 transfer RNAs, and 2 ribosomal RNAs necessary for translation [130]. Similarly, lipids such as phosphotidylethanolamine, phosphotidylglycerine, and cardiolipin are synthesized within the mitochondria from ER-derived phospholipids [131]. The rest of the $\sim 1000$ proteins and lipids come from the nucleus and ER, respectively. This coordinated import and synthesis of proteins and lipids are essential for healthy mitochondrial biogenesis.

Peroxisome proliferator-activated receptor coactivator (PGC-1 $\alpha$ ) is considered to be the master regulator of mitochondrial biogenesis [132]. PGC-1 $\alpha$ is induced under conditions of increased energy demand such as fasting, cold, and exercise where it increases the expression of, and coactivates, a variety of transcription factors [132-135]. These transcription factors include the nuclear respiratory factors (NRF1/2), peroxisome proliferatoractivated receptor (PPAR), as well as estrogen-related receptors (ERRs) [132]. NRF1 and NRF2 promote the expression of the nuclear encoded mitochondrial transcription factor A (Tfam), which is responsible for the transcription of mtDNA [136]. As described previously, mtDNA gives rise to 13 subunits of the electron transport chain as well as the 22 tRNAs and 2 rRNAs. The nuclear proteins come from the transcriptional activity of PPARs and ERRs, which are involved in regulating the expression of proteins and enzymes that control multiple aspects of mitochondrial oxidative metabolism ranging from fatty acid transport and oxidation, glucose utilization, the TCA cycle, to oxidative phosphorylation [137]. Once transcribed in the nucleus, the mRNA is then translated in the cytosol complete with a mitochondrial localization signal. The proteins are subsequently transported in an unfolded fashion with the aid of molecular chaperones such as Hsp70 and inserted into the mitochondria through different protein translocases, including TOM and TIM (both involved in the translocation of PINK1), as well as presequence translocase-associated motor (PAM) and sorting and assembly machinery (SAM) [138]. 
Lipids, on the other hand, are primarily synthesized in the ER and transported to the mitochondria during biogenesis [139]. The transfer of primarily phospholipids [140] from the ER to mitochondria has been thought to be mediated via ER-mitochondrial contacts, effectively termed the mitochondria-associated membranes (MAMs) [141]. The MAMs are purportedly comprised of a variety of proteins including (i) the IP3 receptor and VDAC1, through Grp75, that play a role in calcium signaling; (ii) the mitofusins, expressed both on mitochondria and ER membranes, that play a role in tethering and modulating mitochondrial dynamics; (iii) the ER stress sensor PERK that initiates signaling in response to ER stress; and (iv) many more [142-144]. Although it remains unclear how lipids are transported in MAMs, in yeast, it is thought that ER-mitochondrial encounter structure (ERMES) are responsible [145]. ERMES are composed of the structural component maintenance of mitochondrial morphology 1 (MMM1), mitochondrial distribution and morphology 34 (Mdm34), Mdm12, and Mdm10 as well as the regulatory subunit GTPase EF-hand protein of mitochondria (Gem1) [146]. The ERMES complex possesses a synaptotagminlike mitochondrial lipid-binding (SMP) domain that harbors an elongated hydrophobic groove in which different lipids can bind and possibly be transported [147]. Once the lipids are transported from the ER, mitochondrial enzymes can then synthesize the lipids critical for mitochondrial function.

\section{The Interplay Between Mitochondrial Dynamics and Mitophagy}

Mitochondrial dynamics and mitophagy have been well studied separately, but investigations aimed at elucidating the interplay between these two components of mitochondrial quality control have been limited. It has been shown that fission can trigger mitophagy and govern mitochondrial clearance $[18,148]$. In this regard, multiple studies have demonstrated that alterations to pro-fusion or profission proteins can affect mitophagy, i.e., inhibition of Fis1 in insulin secreting (INS1) cells resulted in a $70 \%$ reduction of mitophagy, while overexpression of Drp1 in HeLa cells was accompanied by a $70 \%$ decrease in mitochondrial mass [148, 149]. Further evidence that fission and mitophagy are intimately associated is that the Drp1dependent mediator of fission, endophilin B1, colocalizes with autophagic markers LC3, Atg5, and Atg9 specifically in response to nutrient starvation [150, 151]. Conversely, proteins associated with mitophagy (in particular, excessive PINK1 in depolarized mitochondria) may also play a role in fission by mechanisms that are, at present, unclear. It has been proposed that when PINK1 accumulates and recruits Parkin, Parkin ubiquitinates mitofusins to inhibit fusion [17]. Accordingly, in a state where all mitochondria are depolarized with PINK1 accumulation and mitofusin ubiquitination, the only path for mitochondria would be fission. However, although fission is apparently necessary for mitophagy, mitophagy is not necessary for fission $[69,92,122,148,152,153]$.

\section{Mitochondrial Quality Control in Disease}

As discussed in the previous sections, mitochondrial dynamics and mitophagy are essential regulators of mitochondrial quality control and play a role in maintaining mitochondrial homeostasis in healthy cells. Defects in mitochondrial quality control have also been implicated to contribute to both chronic and acute neurological and cardiovascular diseases; however, little is known about how mitochondrial dynamics and mitophagy interact/communicate with each other under pathophysiological conditions.

\section{Chronic Diseases: Parkinson's and Alzheimer's Disease}

\section{Parkinson's Disease}

The pathologic signature of $\mathrm{PD}$ is the accumulation-damaged protein aggregates such as $\alpha$-synuclein (SNCA) and ubiquitin into intracytoplasmic inclusions termed Lewy bodies. PD has been associated with mutations, sporadic or hereditary, in at least six genes that are responsible for generating mutations in the following proteins: SNCA, Parkin, $\beta$-glucocerebrosidase (GBA), PINK1, the protein deglycase DJ1, and leucine-rich repeat kinase 2 (LRRK2) [154]. Interestingly, these genes give rise to proteins that are associated with mitochondria or located within mitochondria, thereby implicating mitochondria as key players in PD [155-158]. Evidence of mitochondrial abnormalities in PC (including reduced complex I activity, reduced mitochondrial membrane potential, increased ROS production, altered mitochondrial dynamics, impaired mitochondrial trafficking, and increases in mtDNA mutations) underscore this association [159-163].

To maintain a healthy mitochondrial network, cells must undergo mitophagy to dispose of damaged and dysfunctional mitochondria and produce new healthy mitochondria via mitochondrial biogenesis. In PD, patients with PINK1 and Parkin mutations display impaired mitophagy. Mutations and defects in PINK1 (i) have the potential to diminish both the mitochondrial translocation and activation of Parkin, (ii) can result in the failure to segregate dysfunctional mitochondria for mitophagy via fission [164], and (iii) have been associated with a decrease in phospho-Drp1 levels and an increase in Drp1 GTPase activity, suggesting a direct role of PINK1 to induce fission [165]. PINK1 deficiency has also been shown to be associated with dysfunctional $\mathrm{Na}^{+} / \mathrm{Ca}^{2+}$ exchangers in the inner mitochondrial 
membrane that cause unbalanced mitochondrial calcium homeostasis [160]. This impairment of calcium efflux from the mitochondria results in reduced respiration from ROS-stimulated inhibition of glucose uptake. Finally, and not surprisingly based on the aforementioned associations, mutations in PINK1 reportedly increase the sensitivity of cells to stress-induced cell death. Studies have shown that PINK1 is necessary for long-term survival of cells $[155,166]$.

Mutations in Parkin, on the other hand, can lead to impaired ubiquitination of outer mitochondrial membrane proteins, which has been shown to play a role in recognition by the autophagosome [167]. Interestingly, Parkin was first linked to the mitochondria by evidence that the protein prevented mitochondrial swelling and cytochrome $\mathrm{C}$ release in cells treated with ceramide [168]. In addition to this purported neuroprotective role, Parkin was found to protect mtDNA from oxidative stress and stimulate mtDNA repair systems [169], while, in strains of Parkin knockout mice, neurons in the ventral midbrain displayed severe mitochondrial damage and decreases in complexes I and IV, despite being devoid of the phenotypical motor impairment characteristic of PD [170, 171]. It remains unclear how deficiencies in Parkin lead to severe mitochondrial damage and PD. Mitochondria do undergo a basal level of "wear and tear" via mtDNA mutations as well as oxidation of lipids and proteins. As discussed previously, under normal conditions, these damaged mitochondria would be sequestered and undergo proteolytic degradation. If, in PD, defects in PINK1 and Parkin compromise the ability of the cell to degrade and dispose of proteins or damaged mitochondria, the accrual of damaged organelles would, in all likelihood, ultimately lead to cell death.

\section{Alzheimer's Disease}

AD currently affects 1.5 million Americans, with the associated memory loss and decline in cognitive function attributed to the accumulation of amyloid- $\beta$ plaques and phosphorylated tau [172]. Sporadic and hereditary AD are attributed to mutations in several genes, as well as accumulation of mtDNA mutations that generally lead to an increase in $\beta$-amyloid levels in the brain $[173,174]$. Although the underlying mechanisms are unclear, accumulation of amyloid- $\beta$ in neurons and formation of plaques have been attributed to excessive cleavage of amyloid precursor protein (APP, a transmembrane glycoprotein) or mutations in the apolipoprotein APOE4, which, under normal conditions, contributes to the breakdown of amyloid- $\beta$ [175].

[176].

Early in the pathogenesis of AD, mitochondrial abnormalities are also common, including defective glucose metabolism, a reduction in enzyme activity, mitochondrial DNA mutations, defected gene expression, and aberrant mitochondrial dynamics [177]. Mitochondria in AD patients have been observed to reveal significant structural damage together with decreases in mitochondrial fusion proteins, increases in Fis1, and increases in Ser616 phosphorylated Drp1 (despite decreases in total Drp1) - all of which favor excessive fragmentation $[178,179]$. In vitro studies corroborated this concept, i.e., overexpression of APP in M17 cells was associated with mitochondrial fragmentation, reduced neurite growth, abnormal mitochondrial distribution, and modulation of mitochondrial fission/fusion proteins [180]. Similar findings were obtained in primary neurons of transgenic mice expressing the human APP Swedish mutation [181]. The interactions between amyloid- $\beta$ and Drp1 are still unknown, but limited data have proposed that GSK $3 \beta$ may be the mediator in Drp1 phosphorylation via the association of amyloid- $\beta$ with NMDA receptors and the Wnt signaling pathway [182]. With excessive fission, healthy mitochondria are cleaved unnecessarily, thereby disrupting the equilibration of mitochondrial matrix metabolites (required for efficient production of ATP) and making the mitochondria more vulnerable to injury. In addition, this excessive fragmentation could potentially lead to an upregulation in mitophagic pathways.

\section{Insights into I/R Injury}

Fission, Fusion, and Cell Fate There has been growing interest in mitochondrial dynamics, and its potential association with apoptosis, in the setting of I/R injury [183-185]. Several studies have uncovered excessive mitochondrial fission or fragmentation during both ischemia and I/R injury [186-189]. Using a 6-h OGD model to simulate ischemia, Kim et al. observed a massive mitochondrial fragmentation profile during OGD in H9C2 cells [186]. This mitochondrial fission profile was confirmed in vivo using a 24-h left anterior descending permanent ligation model in mice [186]. Disatnik et al. and Ong et al. observed mitochondrial fragmentation during reoxygenation in OGD/reoxygenation models using neonatal primary cardiomyocytes and HL1 cells, respectively [188, 189]. In the brain, Tang et al. also demonstrated a highly fragmented mitochondrial profile in mouse N2a neuroblastoma cells following OGD/reoxygenation [187]. Previous studies from our lab, conducted using both primary rat neurons and HT22 cells, revealed evidence of mitochondrial fission during OGD and reoxygenation [190]. Moreover, mitochondrial fragmentation was accompanied by Opa1 processing and concomitant release of cytochrome C [190]. Using an in vitro real-time imaging model of OGD/reoxygenation, we further observed complex temporal alterations in mitochondrial morphology [191]. Using HT22 cells transfected with a plasmid containing a GFP marker, two distinct phases of fragmentation were detected: The first phase of fission occurred during OGD, while reintroduction of oxygen triggered initial fusion followed by complete and massive fragmentation after late reoxygenation. The massive fragmentation observed during 
late reoxygenation was confirmed in vivo in CA1 hippocampal neurons of rats exposed to global brain ischemia/ reperfusion [191].

The aforementioned studies suggest that mitochondrial fragmentation is a pathophysiological consequence of $I / R$ injury and that inhibition mitochondrial fragmentation may reverse this. Indeed, in support of this concept, there is evidence that, after exposure to apoptotic stimuli, Drp1 inhibition or overexpression of a dominant negative Drp1 blocked the induction of apoptosis [192]. Moreover, inhibition of Drp1 was found to be neuroprotective in response to OGD in vitro and transient focal ischemia in vivo [193], and cardioprotective in cultured HL-1 cardiomyocytes subjected to OGD and reoxygenation [194]. However, in the latter study, cardioprotection was only seen when inhibition of Drp1 was initiated as a pretreatment; cell death was paradoxically exacerbated when treatment was administered during reoxygenation [194]. This points to the complexity of mitochondrial dynamics and its effects on cell death or survival - an issue that is highlighted by observations that fusion (presumably favoring survival) involves the formation of lipidic pores that may contribute to mitochondrial permeabilization and compromise cell viability [195], while fission (as discussed above, associated with cell death) is necessary for mitophagy and governs clearance of dysfunctional mitochondria [18, 148]. Thus, despite strong evidence to suggest that mitochondrial fragmentation can be detrimental to the cell during stress conditions, collectively these results reveal a complex dynamic nature of mitochondria that requires further study to understand (i) why fission occurs during these stress states, (ii) why inhibition mitochondrial fission is only cardioprotective when initiated before the $\mathrm{OGD} /$ ischemic event, and (iii) why fusion is not possible after reoxygenation/reperfusion.

Mitophagy and I/R Injury I/R injury has been shown to activate mitophagy pathways through multiple signals. During the ischemic phase when ATP production halts, AMPK pathways are upregulated to initiate autophagy [196]. AMPK activates ULK1 via phosphorylation, which will activate the class III PI3K complex (beclin 1, VPS34, and VPS15) that initiate nucleation of the phagophore [197]. Interestingly, ULK1 may have a redundant role in activating mitophagy, i.e., has also been shown to translocate to mitochondria and activate the FUNDC1 receptor [198]. During the reperfusion phase, ROS serves as a signaling molecule to inhibit the mechanistic target of rapamycin (mTOR) pathways, thereby contributing to the initiation and nucleation of the autophagosome [199]. ROS has also been shown to activate mitophagy via BNIP3 although, as stated previously, high levels of BNIP3 can induce apoptosis [200, 201]. Overexpression of BNIP3 in HL-1 myocytes was reported to increase cell death in response to simulated $\mathrm{I} / \mathrm{R}$ injury by facilitating $\mathrm{mPTP}$ opening through the activation of Bcl-2- asscoaited X protein (Bax) $[116,201]$. Moreover, $\mathrm{BNIP}^{-/-}$ mice subjected to 1-h coronary artery occlusion and 3-week reperfusion exhibited preserved left ventricular (LV) systolic function and diminished LV dilation, while conditional overexpression of BNIP3 reversed these effects resulting in increased apoptosis and infarct size [202]. Collectively, these results demonstrate a threshold for BNIP3, as increases or overexpression will inevitably lead to increased apoptosis. Given the dual "life-or-death" role of mitochondria, together with reports of the strong association between mitophagic proteins (i.e., BNIP3 and Drp1) and apoptosis, this raises the question of whether mitophagy is beneficial or detrimental to cell fate in response to $I / R$ injury.

\section{Heart}

During ischemia, upregulation of mitophagy is agreed to confer protection $[203,204]$. The most compelling evidence is provided by Kubli et al. Using an in vivo mouse model, the investigators demonstrated that Parkin-deficient mice are more sensitive to myocardial infarction [204]. Following permanent left anterior descending coronary artery occlusion, Parkin-deficient mice displayed accumulation of swollen and dysfunctional mitochondria due to impaired mitophagy, which resulted in larger infarcts and reduced survival rates [204]. Moreover, the investigators observed upregulation of mitophagy with increased expression of Parkin at the border zone of the infarct in wild-type mice [204]. In vitro studies corroborated this concept, i.e., overexpression of Parkin in isolated cardiomyocytes subjected to hypoxia-mediated cell death was associated with increased Parkin translocation to the mitochondria and increased cell viability, while cardiomyocytes expressing Parkinson disease-associated mutants of Parkin failed to reduce hypoxia-mediated cell death [204]. In accordance with this concept, evidence in the in vivo mouse model of permanent coronary ligation revealed that the upregulation of mitophagy via the genetic deletion of two molecular inhibitors, p53 and TP53-induced glycolysis and apoptosis regulator (TIGAR), attenuated apoptotic cell death and provided resistance to subsequent remodeling [203]. Moreover, cardioprotection was reversed in $\mathrm{p} 53^{-/}$and TIGAR $^{-/-}$mice following permanent myocardial infarction with administration of chloroquine, an autophagy inhibitor, an effect characterized by the accumulation of abnormal mitochondria in the ischemic myocardium [203]. Interestingly, the upregulation of mitophagy via inhibition of p53 and TIGAR was induced through an increase in ROS production followed by BNIP3 activation [203]. In this case, BNIP3 activation was necessary and beneficial in attenuating cardiac I/ $\mathrm{R}$ injury.

In contrast to ischemic injury, the role of mitophagy in $I / R$ injury remains controversial. A considerable body of evidence suggests that an upregulation of mitophagy during myocardial 
$\mathrm{I} / \mathrm{R}$ injury is protective $[118,205-207]$. It was first described that upregulation of autophagy in HL-1 cells protected against simulated ischemia-reperfusion by Hamacher-Brady et al. [207]. The investigator's observations revealed that autophagosomal engulfment of mitochondria was a prominent response in their model. Subsequent studies using HL-1 cells demonstrated an upregulation of BNIP3-regulated mitophagy following simulated I/R [118]. Purportedly, overexpression of BNIP3 during simulated $\mathrm{I} / \mathrm{R}$ induces mitochondrial damage via ROS production, leading to an upregulation of mitophagy [118]. Expression of ATG5K130R, a dominant negative of ATG5 shown to suppress vacuole formation, significantly reduced mitophagy and increased BNIP3-induced cell death [118]. Together, these data suggests that upregulation of mitophagy occurs following BNIP3-induced mitochondrial damage as a cellular response to remove damaged mitochondria during I/R [118]. More recently, using Langendorff heart I/R model, Lu et al. observed that PGAM5-deficient mice had exacerbated necroptosis in response to $25 \mathrm{~min}$ of ischemia followed by $90 \mathrm{~min}$ of reperfusion [205]. Data in their mouse embryonic fibroblast (MEF) model of ROS-dependent necroptosis revealed impaired autophagic removal of LC-3II as well as impaired mitochondrial clearance following $24 \mathrm{~h}$ of TNF- $\alpha$ cyclohexamide and z-VAD-fmk (TCZ) stimulation. Finally, upregulation of mitophagy has been reported to play a role in the gold standard of cardioprotection, ischemic precondition (IPC) [206]. In both Langendorff perfused rat hearts and in vivo mice subjected to regional IPC, Parkin and P62 translocate to the mitochondria and mediate mitophagy [206]. Moreover, IPC is abolished in Parkin-deficient mice, suggesting a critical role for Parkin in IPC [206]. The investigators propose that selective mitophagy of mitochondria that have the lowest threshold for mPTP opening during IPC would leave behind mitochondria that are more equipped to handle sustained ischemic insults [206].

In contrast to the aforementioned studies, there is some evidence to suggest that the suppression of mitophagy may protect the heart from I/R [208]. In a rat model of left anterior descending coronary artery occlusion, pretreatment with mitochondrial aldehyde dehydrogenase 2 (ALDH2), an allosteric tetrameric enzyme responsible for the metabolism or detoxification of toxic aldehydes, conferred cardioprotection via attenuation of apoptotic cell death [208]. In vitro studies corroborated this concept using $\mathrm{H} 9 \mathrm{C} 2$ cells subjected to $2 \mathrm{~h}$ of hypoxia (1\% oxygen) and $1 \mathrm{~h}$ of reoxygenation [208]. Pretreatment of ALDH2 increased cell viability through the suppression of mitophagy [208]. Mitophagy was measured through colocalization of PINK1, Parkin, and the mitochondrial electron transport chain protein cytochrome C oxidase subunit IV (COXIV) [208]. However, given that mitophagy is in constant flux and mitochondria are degraded in autophagolysosomes, mitophagy proteins such as PINK1 and Parkin that are associated with dysfunctional mitochondria would also be degraded. Therefore, evaluation of the mitophagic flux would aid in confirming that ALDH2 is suppressing mitophagy as opposed to enhancing mitophagy and subsequently breakdown of mitophagy proteins.

\section{Brain}

There are notable differences between brain and heart in that controversy lies with respect to the role of mitophagy in ischemia as well as I/R (beneficial or detrimental). Cerebral ischemic preconditioning has been associated with increased autophagosome formation and confers neuroprotection in rats subjected to permanent middle cerebral artery occlusion (pMCAO) [209]. More recent evidence in the same model of pMCAO confirmed that the upregulation of autophagy during ischemia includes mitophagy, revealed by increased autophagic vacuoles containing mitochondria and LC3 colocalization with COXIV [210]. Interestingly, the upregulation of mitophagy was mediated through Drp1 as treatment with Mdivi, a pharmacological inhibitor of Drp1, prevented mitophagy and resulted in decreased LC3 and COXIV colocalization, increased levels of mitochondrial proteins, and the accumulation of damaged mitochondria following $1 \mathrm{~h}$ of pMCAO [210]. Moreover, Drp1 inhibition exacerbated mitochondrial-mediated brain injury to an even greater extent compared to the inhibition of autophagy using 3-methyladenine (3-MA) following $24 \mathrm{~h}$ of pMCAO [210]. Conversely, in mice subjected to pMCAO, administration of 3-MA conferred neuroprotection via a reduction in infarct size along with a dosedependent increase in cell viability following exposure of 4-h OGD in rat primary cortical neurons [211]. Knockdown of Atg7 with siRNA reinforced this concept, resulting in increased cell viability in response to ischemia-induced neuronal injury [210]. Interestingly, the investigators observed no change in infarct volume with administration of mdivi in both in vitro and in vivo models of ischemia [211]. The lack of observable change could be a result of when the inhibitor was administered, i.e., at the point of artery occlusion or the beginning of OGD. Results from our lab demonstrate that mitochondria undergo massive fragmentation within $20 \mathrm{~min}$ of ischemia [191]. In this case, mitochondrial fragmentation could have occurred before the onset of inhibition with mdivi, resulting in segregated mitochondria ready to be recruited for mitophagy.

In cerebral $\mathrm{I} / \mathrm{R}$, there is a consequential amount of literature that suggests that an upregulation of mitophagy confers neuroprotection [205, 211, 212]. The strongest evidence to support this concept is provided by Zhang et al. [211]. Using pharmacological and genetic suppression of autophagy (i.e., 3-MA treatment, bafilomycin A1 (BafA) treatment, Atg5 knockout, and Atg7 knockdown), primary cortical neurons subjected to $2 \mathrm{~h}$ of OGD followed by a 24-h reperfusion had significantly decreased cell viability as compared to controls [211]. These results were affirmed in an in vivo transient MCAO model, where inhibition of autophagy via 3-MA treatment and Atg7 knockdown exacerbated I/R injury [211]. 

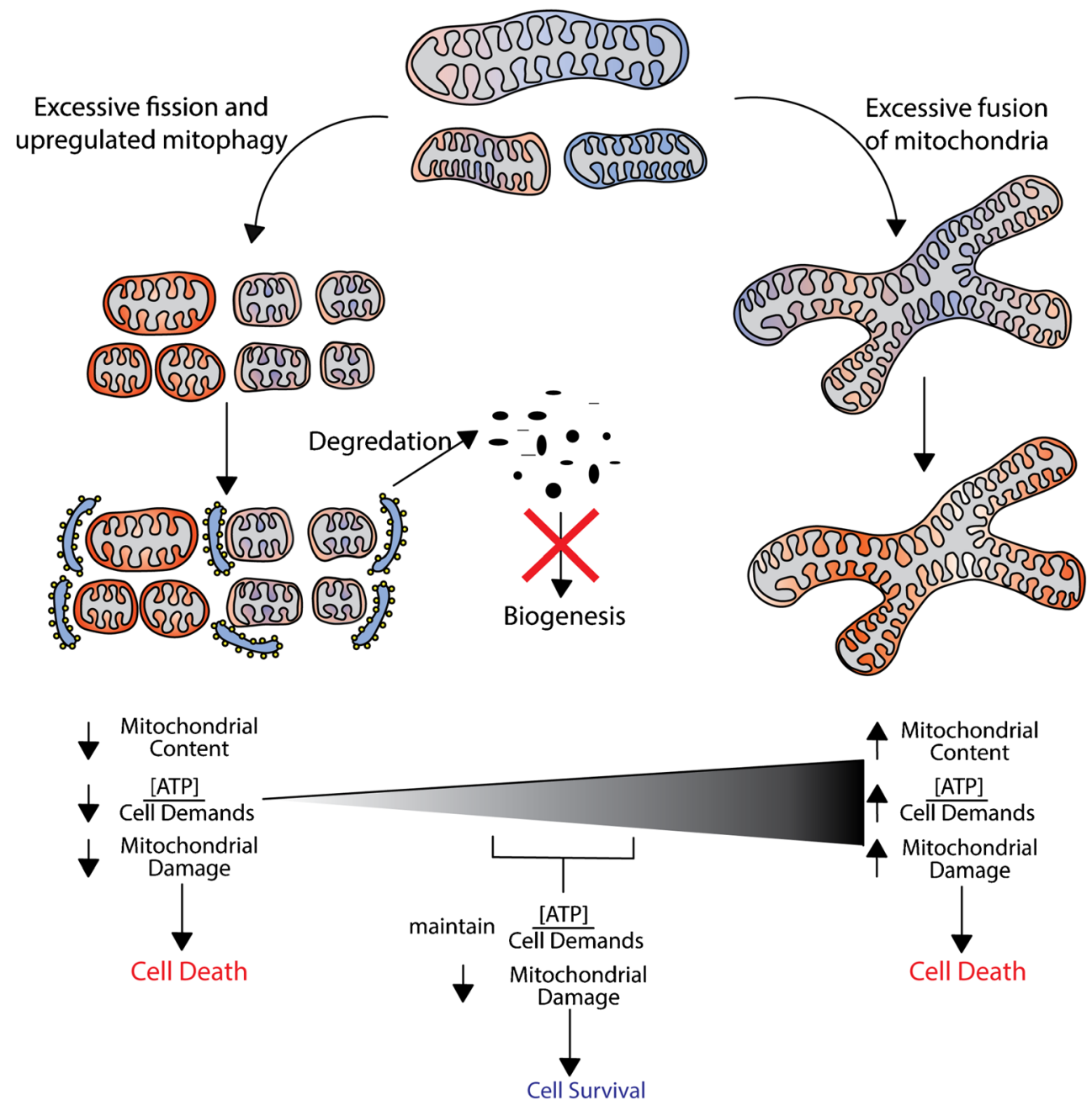

Fig. 5 Finding a balance of mitochondrial quality control. During I/R injury, there is excessive mitochondrial fragmentation, favoring an increase in mitophagy. Degradation of dysfunctional ROS-producing mitochondria is critical for survival; however, mitochondrial content decrease would compromise ATP production. Insufficient ATP production paired with inhibited biogenesis will ultimately lead to cell death. In contrast, if fission was completely inhibited, mitochondrial content would be maintained, but damaged mitochondria would not be segregated and could

Moreover, addition of rapamycin, a known enhancer of mitophagy that attenuates mitochondrial dysfunction following cerebral ischemia [212], partly reversed the deleterious effects of 3-MA-treated primary neurons subjected to OGD reperfusion [211]. Similar to the heart, $\mathrm{PGAM}^{-/}$mice subject to transient MCAO displayed significantly higher infarct sizes as compared to wild-type mice after $72 \mathrm{~h}$ of reperfusion [205].

At variance with the preceding studies, evidence provided by Shi et al. suggests that excessive mitophagy following cerebral I/R results in cell death [213]. Using an adapted Rice-Vanucci model of neonatal stroke or hypoxia-ischemia encephalopathy (HIE), the investigators observed that pups deficient of BNIP3 had a decrease in mitophagy that resulted in significantly smaller infarct sizes in response to neonatal stroke and 7 days of reperfusion [213]. Interestingly, the lead to accumulated mitochondrial dysfunction and exacerbate damage to the entire mitochondrial network. The increase in mitochondrial damage would augment pro-apoptotic stimuli and, ultimately, cause cell death. Therefore, a balance in mitochondrial quality control (i.e., an equilibrium between retaining adequate mitochondrial content for sufficient ATP production versus disposal of dysfunctional mitochondria) is optimal for cell survival after I/R injury

infarct volume in $\mathrm{BNIP} 3^{-/}$pups was significantly larger after 1 day of reperfusion but then recovered while the infarct volume in wild-type pups was exacerbated after 3 to 7 days of reperfusion [213]. The investigators also demonstrated in wild-type pups a dramatic increase in BNIP3 mitochondrial-localized homodimer expression in a timedependent manner following neonatal stroke accompanied by a significant decrease in mitochondrial proteins from isolated cortical neurons following $6 \mathrm{~h}$ of OGD [213]. Although these data were collected in a neonatal model, it suggests that (i) BNIP3 induces excessive mitophagy following $\mathrm{I} / \mathrm{R}$, which ultimately exacerbates cerebral $\mathrm{I} / \mathrm{R}$ injury, and (ii) underscores the balance of mitophagy required to prevent pro-apoptotic proteins such as BNIP3 to surpass the "death signal" threshold. 


\section{Beyond "Good" Versus "Evil": a Question of Balance?}

As reviewed in the preceding sections, there is continued controversy regarding whether upregulation of mitophagy in the setting of $I / R$ is good or bad. It may, however, be more appropriate to consider mitophagy (and mitochondrial quality control as a whole) as a balancing act (Fig. 5). For example, it has been proposed that, following reperfusion, mitophagy is essential to clear dysfunctional mitochondria [211]. However, excessive mitophagy coupled with inhibited mitochondrial biogenesis and a global decrease in protein synthesis $[214,215]$ will result in a decrease in mitochondrial mass and, subsequently, a deficit in ATP production that may fail to meet the demands of the cell. This energy imbalance could eventually cause energy deprivation and cell death. With the other extreme- that is, with minimal mitophagy-damaged mitochondria will not be eliminated and the overall ROS burden would increase. This excessive ROS formation could further induce mitochondrial dysfunction, leading to a feed forward cycle of ROS production and ultimately cell death (Fig. 5).

Accordingly, there is a fundamental need for balance in mitochondrial quality control, and further investigation is needed to define this threshold. If this threshold can be identified, modulation of mitophagy may represent a valuable therapeutic option, with the goal of eliminating dysfunctional mitochondria while still providing sufficient energy to repair cellular damage, restore protein translation, and ultimately return to homeostasis. PINK1 and Parkin (rather than BNIP3 and NIX) may yield the greatest promise as effective targets for manipulation of mitophagy, given their reported favorable association with cell survival together with a lack of involvement in apoptotic pathways [204, 206, 211].

\section{Conclusions and Challenges}

Mitochondrial quality control is critical for the homeostasis of the mitochondrial network, and a constant balance is needed between mitochondrial fission/fusion as well as mitophagy and biogenesis. Disruption of mitochondrial quality control has been proposed to contribute to the pathogenesis of acute and chronic diseases, including Parkinson's disease, Alzheimer's disease, and ischemia-reperfusion-induced cell death in the brain and heart. Accordingly, targeted modulation of one or more of the molecular components involved in mitochondrial quality control provides opportunities for the design of novel therapies. However, to capitalize on this potential opportunity, a greater mechanistic understanding of mitochondrial fission/fusion, mitophagy, and mitochondrial quality control - together with the development improved molecular tools to investigate these complex phenomena [216]—will be required.

Open Access This article is distributed under the terms of the Creative Commons Attribution 4.0 International License (http:// creativecommons.org/licenses/by/4.0/), which permits unrestricted use, distribution, and reproduction in any medium, provided you give appropriate credit to the original author(s) and the source, provide a link to the Creative Commons license, and indicate if changes were made.

\section{References}

1. Mozaffarian D et al (2015) Heart disease and stroke statistics2015 update: a report from the American Heart Association. Circulation 131(4):e29-322

2. Pulsinelli WA, Duffy TE (1983) Regional energy balance in rat brain after transient forebrain ischemia. J Neurochem 40(5):1500 1503

3. Crack PJ, Taylor JM (2005) Reactive oxygen species and the modulation of stroke. Free Radic Biol Med 38(11):1433-1444

4. Aronowski J, Strong R, Grotta JC (1997) Reperfusion injury: demonstration of brain damage produced by reperfusion after transient focal ischemia in rats. J Cereb Blood Flow Metab 17(10): 1048-1056

5. Sanderson TH et al (2013) Molecular mechanisms of ischemiareperfusion injury in brain: pivotal role of the mitochondrial membrane potential in reactive oxygen species generation. Mol Neurobiol 47(1):9-23

6. Lucas DT, Szweda LI (1998) Cardiac reperfusion injury: aging, lipid peroxidation, and mitochondrial dysfunction. Proc Natl Acad Sci U S A 95(2):510-514

7. Alzheimer's A (2016) Alzheimer's disease facts and figures. Alzheimers Dement 2016:12

8. Koller W et al (1987) Relationship of aging to Parkinson's disease. Adv Neurol 45:317-321

9. Turner RS (2006) Alzheimer's disease. Semin Neurol 26(5):499 506

10. Yue R et al (2015) Mitochondrial DNA oxidative damage contributes to cardiomyocyte ischemia/reperfusion-injury in rats: cardioprotective role of lycopene. J Cell Physiol 230(9):21282141

11. Newmeyer DD, Ferguson-Miller S (2003) Mitochondria: releasing power for life and unleashing the machineries of death. Cell 112(4):481-490

12. Anne Stetler R et al (2013) The dynamics of the mitochondrial organelle as a potential therapeutic target. J Cereb Blood Flow Metab 33(1):22-32

13. Ott $\mathrm{M}$ et al (2002) Cytochrome $\mathrm{c}$ release from mitochondria proceeds by a two-step process. Proc Natl Acad Sci U S A 99(3): 1259-1263

14. Twig G, Shirihai OS (2011) The interplay between mitochondrial dynamics and mitophagy. Antioxid Redox Signal 14(10):19391951

15. Calo L et al (2013) Mitochondrial dynamics: an emerging paradigm in ischemia-reperfusion injury. Curr Pharm Des 19(39): 6848-6857

16. Hoppins S, Lackner L, Nunnari J (2007) The machines that divide and fuse mitochondria. Annu Rev Biochem 76:751-780 
17. Twig G, Hyde B, Shirihai OS (2008) Mitochondrial fusion, fission and autophagy as a quality control axis: the bioenergetic view. Biochim Biophys Acta 1777(9):1092-1097

18. Gomes LC, Scorrano L (2008) High levels of Fis1, a pro-fission mitochondrial protein, trigger autophagy. Biochim Biophys Acta 1777(7-8):860-866

19. Malena A et al (2009) Inhibition of mitochondrial fission favours mutant over wild-type mitochondrial DNA. Hum Mol Genet 18(18):3407-3416

20. Suen DF et al (2010) Parkin overexpression selects against a deleterious mtDNA mutation in heteroplasmic cybrid cells. Proc Natl Acad Sci U S A 107(26):11835-11840

21. Partikian A et al (1998) Rapid diffusion of green fluorescent protein in the mitochondrial matrix. J Cell Biol 140(4):821-829

22. Ono T et al (2001) Human cells are protected from mitochondrial dysfunction by complementation of DNA products in fused mitochondria. Nat Genet 28(3):272-275

23. Busch KB et al (2006) Mitochondrial dynamics generate equal distribution but patchwork localization of respiratory complex I. Mol Membr Biol 23(6):509-520

24. Dimmer KS, Scorrano L (2006) (De) constructing mitochondria: what for? Physiology (Bethesda):233-241

25. Song BD, Schmid SL (2003) A molecular motor or a regulator? Dynamin's in a class of its own. Biochemistry 42(6):1369-1376

26. Boldogh IR, Pon LA (2006) Interactions of mitochondria with the actin cytoskeleton. Biochim Biophys Acta 1763(5-6):450-462

27. Hollenbeck PJ, Saxton WM (2005) The axonal transport of mitochondria. J Cell Sci 118(Pt 23):5411-5419

28. Smirnova E et al (2001) Dynamin-related protein Drp1 is required for mitochondrial division in mammalian cells. Mol Biol Cell 12(8):2245-2256

29. Labrousse AM et al (1999) C. elegans dynamin-related protein DRP-1 controls severing of the mitochondrial outer membrane. Mol Cell 4(5):815-826

30. De Vos KJ et al (2005) Mitochondrial function and actin regulate dynamin-related. Protein 1-dependent mitochondrial fission. Curr Biol 15(7):678-683

31. Varadi A et al (2004) Cytoplasmic dynein regulates the subcellular distribution of mitochondria by controlling the recruitment of the fission factor dynamin-related protein-1. J Cell Sci 117(Pt 19): 4389-4400

32. Jahani-Asl A, Slack RS (2007) The phosphorylation state of Drp1 determines cell fate. EMBO Rep 8(10):912-913

33. Cribbs JT, Strack S (2007) Reversible phosphorylation of Drp1 by cyclic AMP-dependent protein kinase and calcineurin regulates mitochondrial fission and cell death. EMBO Rep 8(10):939-944

34. Wang $\mathrm{W}$ et al (2012) Mitochondrial fission triggered by hyperglycemia is mediated by ROCK1 activation in podocytes and endothelial cells. Cell Metab 15(2):186-200

35. Han XJ et al (2008) CaM kinase I alpha-induced phosphorylation of Drp1 regulates mitochondrial morphology. J Cell Biol 182(3):573-585

36. Palmer CS et al (2011) MiD49 and MiD51, new components of the mitochondrial fission machinery. EMBO Rep 12(6):565-573

37. Osellame LD et al (2016) Cooperative and independent roles of the Drp1 adaptors Mff, MiD49 and MiD51 in mitochondrial fission. J Cell Sci 129(11):2170-2181

38. Stojanovski D et al (2004) Levels of human Fis1 at the mitochondrial outer membrane regulate mitochondrial morphology. J Cell Sci 117(Pt 7):1201-1210

39. Yoon $\mathrm{Y}$ et al (2003) The mitochondrial protein $\mathrm{hFis} 1$ regulates mitochondrial fission in mammalian cells through an interaction with the dynamin-like protein DLP1. Mol Cell Biol 23(15):5409-5420
40. Zhang Y et al (2012) Crystal structure of mitochondrial fission complex reveals scaffolding function for mitochondrial division 1 (Mdv1) coiled coil. J Biol Chem 287(13):9855-9861

41. Otera $\mathrm{H}$ et al (2010) Mff is an essential factor for mitochondrial recruitment of Drp1 during mitochondrial fission in mammalian cells. J Cell Biol 191(6):1141-1158

42. Loson OC et al (2013) Fis1, Mff, MiD49, and MiD51 mediate Drp1 recruitment in mitochondrial fission. Mol Biol Cell 24(5): 659-667

43. Lee H, Yoon Y (2014) Mitochondrial fission: regulation and ER connection. Mol Cells 37(2):89-94

44. Taguchi N et al (2007) Mitotic phosphorylation of dynaminrelated GTPase Drp1 participates in mitochondrial fission. J Biol Chem 282(15):11521-11529

45. Yu T, Jhun BS, Yoon Y (2011) High-glucose stimulation increases reactive oxygen species production through the calcium and mitogen-activated protein kinase-mediated activation of mitochondrial fission. Antioxid Redox Signal 14(3):425-437

46. Qi X et al (2011) Aberrant mitochondrial fission in neurons induced by protein kinase $\mathrm{C}$ \{delta\} under oxidative stress conditions in vivo. Mol Biol Cell 22(2):256-265

47. Chang CR, Blackstone C (2007) Cyclic AMP-dependent protein kinase phosphorylation of Drp1 regulates its GTPase activity and mitochondrial morphology. J Biol Chem 282(30):21583-21587

48. Slupe AM et al (2013) A calcineurin docking motif (LXVP) in dynamin-related. Protein 1 contributes to mitochondrial fragmentation and ischemic neuronal injury. J Biol Chem 288(17):1235312365

49. Wang JX et al (2011) miR-499 regulates mitochondrial dynamics by targeting calcineurin and dynamin-related protein-1. Nat Med 17(1):71-78

50. Friedman JR et al (2011) ER tubules mark sites of mitochondrial division. Science 334(6054):358-362

51. Korobova F, Ramabhadran V, Higgs HN (2013) An actindependent step in mitochondrial fission mediated by the ERassociated formin INF2. Science 339(6118):464-467

52. Santel A, Fuller MT (2001) Control of mitochondrial morphology by a human mitofusin. J Cell Sci 114(Pt 5):867-874

53. Escobar-Henriques M, Anton F (2013) Mechanistic perspective of mitochondrial fusion: tubulation vs. fragmentation. Biochim Biophys Acta 1833(1):162-175

54. Ishihara $\mathrm{N}$ et al (2006) Regulation of mitochondrial morphology through proteolytic cleavage of OPA1. EMBO J 25(13):29662977

55. Chernomordik LV, Kozlov MM (2005) Membrane hemifusion: crossing a chasm in two leaps. Cell 123(3):375-382

56. Meglei G, McQuibban GA (2009) The dynamin-related protein Mgm1 $\mathrm{p}$ assembles into oligomers and hydrolyzes GTP to function in mitochondrial membrane fusion. Biochemistry 48(8):1774 1784

57. Olichon A et al (2007) OPA1 alternate splicing uncouples an evolutionary conserved function in mitochondrial fusion from a vertebrate restricted function in apoptosis. Cell Death Differ 14(4):682-692

58. Delettre $C$ et al (2001) Mutation spectrum and splicing variants in the OPA1 gene. Hum Genet 109(6):584-591

59. Kaser $M$ et al (2003) Oma1, a novel membrane-bound metallopeptidase in mitochondria with activities overlapping with the m-AAA protease. J Biol Chem 278(47):46414-46423

60. MacVicar T, Langer T (2016) OPA1 processing in cell death and disease - the long and short of it. J Cell Sci 129(12):2297-2306

61. Griparic L, Kanazawa T, van der Bliek AM (2007) Regulation of the mitochondrial dynamin-like protein Opal by proteolytic cleavage. J Cell Biol 178(5):757-764 
62. Song $\mathrm{Z}$ et al (2007) OPA1 processing controls mitochondrial fusion and is regulated by mRNA splicing, membrane potential, and Yme1L. J Cell Biol 178(5):749-755

63. Anand $\mathrm{R}$ et al (2014) The i-AAA protease YME1L and OMA1 cleave OPA1 to balance mitochondrial fusion and fission. J Cell Biol 204(6):919-929

64. Ehses S et al (2009) Regulation of OPA1 processing and mitochondrial fusion by m-AAA protease isoenzymes and OMA1. J Cell Biol 187(7):1023-1036

65. Head B et al (2009) Inducible proteolytic inactivation of OPA1 mediated by the OMA1 protease in mammalian cells. J Cell Biol 187(7):959-966

66. Wai T, Langer T (2016) Mitochondrial dynamics and metabolic regulation. Trends Endocrinol Metab 27(2):105-117

67. Baricault L et al (2007) OPA1 cleavage depends on decreased mitochondrial ATP level and bivalent metals. Exp Cell Res 313(17):3800-3808

68. Merkwirth C et al (2008) Prohibitins control cell proliferation and apoptosis by regulating OPA1-dependent cristae morphogenesis in mitochondria. Genes Dev 22(4):476-488

69. Tanaka A et al (2010) Proteasome and p97 mediate mitophagy and degradation of mitofusins induced by Parkin. J Cell Biol 191(7): $1367-1380$

70. Nakatogawa $\mathrm{H}$ et al (2009) Dynamics and diversity in autophagy mechanisms: lessons from yeast. Nat Rev Mol Cell Biol 10(7):458-467

71. Yang Z, Klionsky DJ (2010) Eaten alive: a history of macroautophagy. Nat Cell Biol 12(9):814-822

72. Sharifi MN et al (2015) Measuring autophagy in stressed cells. Methods Mol Biol 1292:129-150

73. Glick D, Barth S, Macleod KF (2010) Autophagy: cellular and molecular mechanisms. J Pathol 221(1):3-12

74. Saftig P, Beertsen W, Eskelinen EL (2008) LAMP-2: a control step for phagosome and autophagosome maturation. Autophagy 4(4): $510-512$

75. Klionsky DJ, Schulman BA (2014) Dynamic regulation of macroautophagy by distinctive ubiquitin-like proteins. Nat Struct Mol Biol 21(4):336-345

76. Feng Y et al (2014) The machinery of macroautophagy. Cell Res 24(1):24-41

77. Nah J, Yuan J, Jung YK (2015) Autophagy in neurodegenerative diseases: from mechanism to therapeutic approach. Mol Cells 38(5):381-389

78. Moyzis AG, Sadoshima J, Gustafsson AB (2015) Mending a broken heart: the role of mitophagy in cardioprotection. Am J Physiol Heart Circ Physiol 308(3):H183-H192

79. Russell RC et al (2013) ULK1 induces autophagy by phosphorylating Beclin-1 and activating VPS34 lipid kinase. Nat Cell Biol 15(7):741-750

80. Lamark T et al (2009) NBR1 and p62 as cargo receptors for selective autophagy of ubiquitinated targets. Cell Cycle 8(13): 1986-1990

81. Zhang J, Ney PA (2009) Role of BNIP3 and NIX in cell death, autophagy, and mitophagy. Cell Death Differ 16(7):939-946

82. Tanida I, Ueno T, Kominami E (2008) LC3 and autophagy. Methods Mol Biol 445:77-88

83. Nair U et al (2011) SNARE proteins are required for macroautophagy. Cell 146(2):290-302

84. Razi M, Chan EY, Tooze SA (2009) Early endosomes and endosomal coatomer are required for autophagy. J Cell Biol 185(2):305-321

85. Rusten TE et al (2007) ESCRTs and Fab1 regulate distinct steps of autophagy. Curr Biol 17(20):1817-1825

86. Nickerson DP, Brett CL, Merz AJ (2009) Vps-C complexes: gatekeepers of endolysosomal traffic. Curr Opin Cell Biol 21(4):543-551
87. Eskelinen EL et al (2002) Role of LAMP-2 in lysosome biogenesis and autophagy. Mol Biol Cell 13(9):3355-3368

88. Jager $\mathrm{S}$ et al (2004) Role for Rab7 in maturation of late autophagic vacuoles. J Cell Sci 117(Pt 20):4837-4848

89. Matsunaga $\mathrm{K}$ et al (2009) Two Beclin 1-binding proteins, Atg14L and Rubicon, reciprocally regulate autophagy at different stages. Nat Cell Biol 11(4):385-396

90. Leu JI et al (2009) A small molecule inhibitor of inducible heat shock protein 70. Mol Cell 36(1):15-27

91. Chen D et al (2012) A mammalian autophagosome maturation mechanism mediated by TECPR 1 and the Atg12-Atg 5 conjugate. Mol Cell 45(5):629-641

92. Narendra DP et al (2010) PINK1 is selectively stabilized on impaired mitochondria to activate Parkin. PLoS Biol 8(1):e1000298

93. Matsuda $\mathrm{N}$ et al (2010) PINK1 stabilized by mitochondrial depolarization recruits Parkin to damaged mitochondria and activates latent Parkin for mitophagy. J Cell Biol 189(2):211-221

94. Mokranjac D, Neupert W (2007) Protein import into isolated mitochondria. Methods Mol Biol 372:277-286

95. Meissner $\mathrm{C}$ et al (2011) The mitochondrial intramembrane protease PARL cleaves human Pink1 to regulate Pink1 trafficking. J Neurochem 117(5):856-867

96. Jin SM et al (2010) Mitochondrial membrane potential regulates PINK1 import and proteolytic destabilization by PARL. J Cell Biol 191(5):933-942

97. Kane LA et al (2014) PINK1 phosphorylates ubiquitin to activate Parkin E3 ubiquitin ligase activity. J Cell Biol 205(2):143-153

98. Ordureau A et al (2015) Defining roles of PARKIN and ubiquitin phosphorylation by PINK1 in mitochondrial quality control using a ubiquitin replacement strategy. Proc Natl Acad Sci U S A 112(21):6637-6642

99. Kazlauskaite A et al (2014) Parkin is activated by PINK1dependent phosphorylation of ubiquitin at Ser65. Biochem J 460(1):127-139

100. Koyano F et al (2014) Ubiquitin is phosphorylated by PINK1 to activate parkin. Nature 510(7503):162-166

101. Okatsu K et al (2015) Phosphorylated ubiquitin chain is the genuine Parkin receptor. J Cell Biol 209(1):111-128

102. Sha D, Chin LS, Li L (2010) Phosphorylation of parkin by Parkinson disease-linked kinase PINK1 activates parkin E3 ligase function and NF-kappaB signaling. Hum Mol Genet 19(2):352-363

103. Kondapalli $\mathrm{C}$ et al (2012) PINK1 is activated by mitochondrial membrane potential depolarization and stimulates Parkin E3 ligase activity by phosphorylating serine 65. Open Biol 2(5):120080

104. Ordureau A et al (2014) Quantitative proteomics reveal a feedforward mechanism for mitochondrial PARKIN translocation and ubiquitin chain synthesis. Mol Cell 56(3):360-375

105. Chan NC et al (2011) Broad activation of the ubiquitinproteasome system by Parkin is critical for mitophagy. Hum Mol Genet 20(9):1726-1737

106. Geisler S et al (2010) PINK1/Parkin-mediated mitophagy is dependent on VDAC1 and p62/SQSTM1. Nat Cell Biol 12(2):119-131

107. Kishore $\mathrm{N}$ et al (2002) IKK-i and TBK-1 are enzymatically distinct from the homologous enzyme IKK-2: comparative analysis of recombinant human IKK-i, TBK-1, and IKK-2. J Biol Chem 277(16):13840-13847

108. Heo JM et al (2015) The PINK1-PARKIN mitochondrial ubiquitylation pathway drives a program of OPTN/NDP52 recruitment and TBK1 activation to promote mitophagy. Mol Cell 60(1):7-20

109. Lazarou $\mathrm{M}$ et al (2015) The ubiquitin kinase PINK1 recruits autophagy receptors to induce mitophagy. Nature 524(7565):309-314 
110. Shen WC et al (2015) Mutations in the ubiquitin-binding domain of OPTN/optineurin interfere with autophagy-mediated degradation of misfolded proteins by a dominant-negative mechanism. Autophagy 11(4):685-700

111. Wild P et al (2011) Phosphorylation of the autophagy receptor optineurin restricts Salmonella growth. Science 333(6039):228-233

112. von Muhlinen N et al (2012) LC3C, bound selectively by a noncanonical LIR motif in NDP52, is required for antibacterial autophagy. Mol Cell 48(3):329-342

113. Thurston TL et al (2009) The TBK1 adaptor and autophagy receptor NDP52 restricts the proliferation of ubiquitin-coated bacteria. Nat Immunol 10(11):1215-1221

114. Lee JY et al (2010) Disease-causing mutations in parkin impair mitochondrial ubiquitination, aggregation, and HDAC6dependent mitophagy. J Cell Biol 189(4):671-679

115. Lee JY et al (2010) HDAC6 controls autophagosome maturation essential for ubiquitin-selective quality-control autophagy. EMBO J 29(5):969-980

116. Kubli DA, Ycaza JE, Gustafsson AB (2007) Bnip3 mediates mitochondrial dysfunction and cell death through Bax and Bak. Biochem J 405(3):407-415

117. Yussman MG et al (2002) Mitochondrial death protein Nix is induced in cardiac hypertrophy and triggers apoptotic cardiomyopathy. Nat Med 8(7):725-730

118. Hamacher-Brady A et al (2007) Response to myocardial ischemia/ reperfusion injury involves Bnip3 and autophagy. Cell Death Differ 14(1):146-157

119. Sandoval H et al (2008) Essential role for Nix in autophagic maturation of erythroid cells. Nature 454(7201):232-235

120. Novak I et al (2010) Nix is a selective autophagy receptor for mitochondrial clearance. EMBO Rep 11(1):45-51

121. Rikka S et al (2011) Bnip3 impairs mitochondrial bioenergetics and stimulates mitochondrial turnover. Cell Death Differ 18(4):721-731

122. Lee $Y$ et al (2011) Mitochondrial autophagy by Bnip3 involves Drp1-mediated mitochondrial fission and recruitment of Parkin in cardiac myocytes. Am J Physiol Heart Circ Physiol 301(5): H1924-H1931

123. Liu L et al (2012) Mitochondrial outer-membrane protein FUNDC1 mediates hypoxia-induced mitophagy in mammalian cells. Nat Cell Biol 14(2):177-185

124. Chen $\mathrm{G}$ et al (2014) A regulatory signaling loop comprising the PGAM5 phosphatase and CK2 controls receptor-mediated mitophagy. Mol Cell 54(3):362-377

125. Lu W et al (2014) Genetic deficiency of the mitochondrial protein PGAM5 causes a Parkinson's-like movement disorder. Nat Commun 5:4930

126. Tracy $\mathrm{K}$ et al (2007) BNIP3 is an RB/E2F target gene required for hypoxia-induced autophagy. Mol Cell Biol 27(17):6229-6242

127. Houtkooper RH, Vaz FM (2008) Cardiolipin, the heart of mitochondrial metabolism. Cell Mol Life Sci 65(16):2493-2506

128. Chu CT et al (2013) Cardiolipin externalization to the outer mitochondrial membrane acts as an elimination signal for mitophagy in neuronal cells. Nat Cell Biol 15(10):1197-1205

129. Kagan VE et al (2016) NDPK-D (NM23-H4)-mediated externalization of cardiolipin enables elimination of depolarized mitochondria by mitophagy. Cell Death Differ 23(7):1140-1151

130. Taanman JW (1999) The mitochondrial genome: structure, transcription, translation and replication. Biochim Biophys Acta 1410(2):103-123

131. Tatsuta T, Scharwey M, Langer T (2014) Mitochondrial lipid trafficking. Trends Cell Biol 24(1):44-52

132. Wu Z et al (1999) Mechanisms controlling mitochondrial biogenesis and respiration through the thermogenic coactivator PGC-1. Cell 98(1):115-124
133. Nisoli E et al (2005) Calorie restriction promotes mitochondrial biogenesis by inducing the expression of eNOS. Science 310(5746):314-317

134. Puigserver $P$ et al (1998) A cold-inducible coactivator of nuclear receptors linked to adaptive thermogenesis. Cell 92(6):829-839

135. Zong $\mathrm{H}$ et al (2002) AMP kinase is required for mitochondrial biogenesis in skeletal muscle in response to chronic energy deprivation. Proc Natl Acad Sci U S A 99(25):15983-15987

136. Virbasius JV, Scarpulla RC (1994) Activation of the human mitochondrial transcription factor A gene by nuclear respiratory factors: a potential regulatory link between nuclear and mitochondrial gene expression in organelle biogenesis. Proc Natl Acad Sci U S A 91(4):1309-1313

137. Fan W, Evans R (2015) PPARs and ERRs: molecular mediators of mitochondrial metabolism. Curr Opin Cell Biol 33:49-54

138. Wiedemann N, Frazier AE, Pfanner N (2004) The protein import machinery of mitochondria. J Biol Chem 279(15):14473-14476

139. Lev S (2012) Nonvesicular lipid transfer from the endoplasmic reticulum. Cold Spring Harb Perspect Biol 4(10)

140. Fleischer $\mathrm{S}$ et al (1967) Lipid composition of mitochondria from bovine heart, liver, and kidney. J Lipid Res 8(3):170-180

141. Gaigg B et al (1995) Characterization of a microsomal subfraction associated with mitochondria of the yeast, Saccharomyces cerevisiae. Involvement in synthesis and import of phospholipids into mitochondria. Biochim Biophys Acta 1234(2):214-220

142. de Brito OM, Scorrano L (2008) Mitofusin 2 tethers endoplasmic reticulum to mitochondria. Nature 456(7222):605-610

143. Szabadkai G et al (2006) Chaperone-mediated coupling of endoplasmic reticulum and mitochondrial $\mathrm{Ca} 2+$ channels. J Cell Biol 175(6):901-911

144. Verfaillie T et al (2012) PERK is required at the ER-mitochondrial contact sites to convey apoptosis after ROS-based ER stress. Cell Death Differ 19(11):1880-1891

145. Kornmann B (2013) The molecular hug between the ER and the mitochondria. Curr Opin Cell Biol 25(4):443-448

146. Boldogh IR et al (2003) A protein complex containing Mdm10p, $\mathrm{Mdm} 12 \mathrm{p}$, and Mmm1p links mitochondrial membranes and DNA to the cytoskeleton-based segregation machinery. Mol Biol Cell 14(11):4618-4627

147. Kopec KO, Alva V, Lupas AN (2010) Homology of SMP domains to the TULIP superfamily of lipid-binding proteins provides a structural basis for lipid exchange between ER and mitochondria. Bioinformatics 26(16):1927-1931

148. Twig G et al (2008) Fission and selective fusion govern mitochondrial segregation and elimination by autophagy. EMBO J 27(2): 433-446

149. Arnoult D et al (2005) Bax/Bak-dependent release of DDP/ TIMM8a promotes Drp1-mediated mitochondrial fission and mitoptosis during programmed cell death. Curr Biol 15(23): 2112-2118

150. Takahashi Y et al (2007) Bif-1 interacts with Beclin 1 through UVRAG and regulates autophagy and tumorigenesis. Nat Cell Biol 9(10):1142-1151

151. Karbowski M, Jeong SY, Youle RJ (2004) Endophilin B1 is required for the maintenance of mitochondrial morphology. J Cell Biol 166(7):1027-1039

152. Frank $\mathrm{M}$ et al (2012) Mitophagy is triggered by mild oxidative stress in a mitochondrial fission dependent manner. Biochim Biophys Acta 1823(12):2297-2310

153. Prieto $J$ et al (2016) Early ERK1/2 activation promotes DRP1dependent mitochondrial fission necessary for cell reprogramming. Nat Commun 7:11124

154. Alcalay RN et al (2010) Frequency of known mutations in earlyonset Parkinson disease: implication for genetic counseling: the consortium on risk for early onset Parkinson disease study. Arch Neurol 67(9):1116-1122 
155. Valente EM et al (2004) Hereditary early-onset Parkinson's disease caused by mutations in PINK1. Science 304(5674): $1158-1160$

156. Biskup $\mathrm{S}$ et al (2006) Localization of LRRK2 to membranous and vesicular structures in mammalian brain. Ann Neurol 60(5):557-569

157. Junn E et al (2009) Mitochondrial localization of DJ-1 leads to enhanced neuroprotection. J Neurosci Res 87(1):123-129

158. Shavali S et al (2008) Mitochondrial localization of alphasynuclein protein in alpha-synuclein overexpressing cells. Neurosci Lett 439(2):125-128

159. Schapira AH (1993) Mitochondrial complex I deficiency in Parkinson's disease. Adv Neurol 60:288-291

160. Gandhi S et al (2009) PINK1-associated Parkinson's disease is caused by neuronal vulnerability to calcium-induced cell death. Mol Cell 33(5):627-638

161. Poole AC et al (2008) The PINK1/Parkin pathway regulates mitochondrial morphology. Proc Natl Acad Sci U S A 105(5):16381643

162. Weihofen A et al (2009) Pink1 forms a multiprotein complex with Miro and Milton, linking Pink1 function to mitochondrial trafficking. Biochemistry 48(9):2045-2052

163. Luoma $\mathrm{P}$ et al (2004) Parkinsonism, premature menopause, and mitochondrial DNA polymerase gamma mutations: clinical and molecular genetic study. Lancet 364(9437):875-882

164. Deng H et al (2008) The Parkinson's disease genes pink1 and parkin promote mitochondrial fission and/or inhibit fusion in Drosophila. Proc Natl Acad Sci U S A 105(38):14503-14508

165. Sandebring A et al (2009) Mitochondrial alterations in PINK1 deficient cells are influenced by calcineurin-dependent dephosphorylation of dynamin-related protein 1. PLoS One 4(5):e5701

166. Wood-Kaczmar A et al (2008) PINK1 is necessary for long term survival and mitochondrial function in human dopaminergic neurons. PLoS One 3(6):e2455

167. Kirkin V et al (2009) A role for ubiquitin in selective autophagy. Mol Cell 34(3):259-269

168. Darios F et al (2003) Parkin prevents mitochondrial swelling and cytochrome $\mathrm{c}$ release in mitochondria-dependent cell death. Hum Mol Genet 12(5):517-526

169. Rothfuss $\mathrm{O}$ et al (2009) Parkin protects mitochondrial genome integrity and supports mitochondrial DNA repair. Hum Mol Genet 18(20):3832-3850

170. Stichel CC et al (2007) Mono- and double-mutant mouse models of Parkinson's disease display severe mitochondrial damage. Hum Mol Genet 16(20):2377-2393

171. Palacino JJ et al (2004) Mitochondrial dysfunction and oxidative damage in parkin-deficient mice. J Biol Chem 279(18):18614 18622

172. Nalls MA et al (2015) Diagnosis of Parkinson's disease on the basis of clinical and genetic classification: a population-based modelling study. Lancet Neurol 14(10):1002-1009

173. Waring SC, Rosenberg RN (2008) Genome-wide association studies in Alzheimer disease. Arch Neurol 65(3):329-334

174. Yan MH, Wang X, Zhu X (2013) Mitochondrial defects and oxidative stress in Alzheimer disease and Parkinson disease. Free Radic Biol Med 62:90-101

175. Wilquet V, De Strooper B (2004) Amyloid-beta precursor protein processing in neurodegeneration. Curr Opin Neurobiol 14(5):582-588

176. Liu CC et al (2013) Apolipoprotein E and Alzheimer disease: risk, mechanisms and therapy. Nat Rev Neurol 9(2):106-118

177. Reddy $\mathrm{PH}$ et al (2012) Abnormal mitochondrial dynamics and synaptic degeneration as early events in Alzheimer's disease: implications to mitochondria-targeted antioxidant therapeutics. Biochim Biophys Acta 1822(5):639-649
178. Hirai K et al (2001) Mitochondrial abnormalities in Alzheimer's disease. J Neurosci 21(9):3017-3023

179. Wang $X$ et al (2009) Impaired balance of mitochondrial fission and fusion in Alzheimer's disease. J Neurosci 29(28):9090-9103

180. Wang $\mathrm{X}$ et al (2008) Amyloid-beta overproduction causes abnormal mitochondrial dynamics via differential modulation of mitochondrial fission/fusion proteins. Proc Natl Acad Sci U S A 105(49):19318-19323

181. Calkins MJ et al (2011) Impaired mitochondrial biogenesis, defective axonal transport of mitochondria, abnormal mitochondrial dynamics and synaptic degeneration in a mouse model of Alzheimer's disease. Hum Mol Genet 20(23):4515-4529

182. Kandimalla R, Reddy PH (2016) Multiple faces of dynamin-related. Protein 1 and its role in Alzheimer's disease pathogenesis. Biochim Biophys Acta 1862(4):814-828

183. Tanaka A, Youle RJ (2008) A chemical inhibitor of DRP1 uncouples mitochondrial fission and apoptosis. Mol Cell 29(4):409-410

184. Martinou JC, Youle RJ (2006) Which came first, the cytochrome c release or the mitochondrial fission? Cell Death Differ 13(8): 1291-1295

185. Estaquier J, Arnoult D (2007) Inhibiting Drp1-mediated mitochondrial fission selectively prevents the release of cytochrome $\mathrm{c}$ during apoptosis. Cell Death Differ 14(6):1086-1094

186. Kim H et al (2011) Fine-tuning of Drp1/Fis1 availability by AKAP121/Siah 2 regulates mitochondrial adaptation to hypoxia. Mol Cell 44(4):532-544

187. Tang $\mathrm{J}$ et al (2016) Parkin protects against oxygen-glucose deprivation/reperfusion insult by promoting Drp1 degradation. Oxidative Med Cell Longev 2016:8474303

188. Ong SB et al (2010) Inhibiting mitochondrial fission protects the heart against ischemia/reperfusion injury. Circulation 121(18): 2012-2022

189. Disatnik MH et al (2013) Acute inhibition of excessive mitochondrial fission after myocardial infarction prevents long-term cardiac dysfunction. J Am Heart Assoc 2(5):e000461

190. Sanderson TH, Raghunayakula S, Kumar R (2015) Neuronal hypoxia disrupts mitochondrial fusion. Neuroscience 301:71-78

191. Kumar R et al (2016) Mitochondrial dynamics following global cerebral ischemia. Mol Cell Neurosci 76:68-75

192. Frank S et al (2001) The role of dynamin-related protein 1, a mediator of mitochondrial fission, in apoptosis. Dev Cell 1(4): $515-525$

193. Grohm J et al (2012) Inhibition of Drp1 provides neuroprotection in vitro and in vivo. Cell Death Differ 19(9):1446-1458

194. Dong Y, Undyala VV, Przyklenk K (2016) Inhibition of mitochondrial fission as a molecular target for cardioprotection: critical importance of the timing of treatment. Basic Res Cardiol 111(5):59

195. Papanicolaou KN, Phillippo MM, Walsh K (2012) Mitofusins and the mitochondrial permeability transition: the potential downside of mitochondrial fusion. Am J Physiol Heart Circ Physiol 303(3): $\mathrm{H} 243-\mathrm{H} 255$

196. Zaha VG, Young LH (2012) AMP-activated protein kinase regulation and biological actions in the heart. Circ Res 111(6):800-814

197. Kim J et al (2011) AMPK and mTOR regulate autophagy through direct phosphorylation of Ulk1. Nat Cell Biol 13(2):132-141

198. Kundu M et al (2008) Ulk1 plays a critical role in the autophagic clearance of mitochondria and ribosomes during reticulocyte maturation. Blood 112(4):1493-1502

199. Alexander A et al (2010) ATM signals to TSC2 in the cytoplasm to regulate mTORC1 in response to ROS. Proc Natl Acad Sci U S A 107(9):4153-4158

200. Scherz-Shouval R et al (2007) Reactive oxygen species are essential for autophagy and specifically regulate the activity of Atg4. EMBO J 26(7):1749-1760 
201. Kubli DA et al (2008) Bnip3 functions as a mitochondrial sensor of oxidative stress during myocardial ischemia and reperfusion. Am J Physiol Heart Circ Physiol 295(5):H2025-H2031

202. Diwan A et al (2007) Inhibition of ischemic cardiomyocyte apoptosis through targeted ablation of Bnip3 restrains postinfarction remodeling in mice. J Clin Invest 117(10):2825-2833

203. Hoshino A et al (2012) p53-TIGAR axis attenuates mitophagy to exacerbate cardiac damage after ischemia. J Mol Cell Cardiol 52(1):175-184

204. Kubli DA et al (2013) Parkin protein deficiency exacerbates cardiac injury and reduces survival following myocardial infarction. J Biol Chem 288(2):915-926

205. Lu W et al (2016) Mitochondrial protein PGAM5 regulates mitophagic protection against cell necroptosis. PLoS One 11(1): e0147792

206. Huang C et al (2011) Preconditioning involves selective mitophagy mediated by Parkin and p62/SQSTM1. PLoS One 6(6):e20975

207. Hamacher-Brady A, Brady NR, Gottlieb RA (2006) Enhancing macroautophagy protects against ischemia/reperfusion injury in cardiac myocytes. J Biol Chem 281(40):29776-29787

208. Ji W et al (2016) Aldehyde dehydrogenase 2 has cardioprotective effects on myocardial ischaemia/reperfusion injury via suppressing mitophagy. Front Pharmacol 7:101
209. Sheng R et al (2010) Autophagy activation is associated with neuroprotection in a rat model of focal cerebral ischemic preconditioning. Autophagy 6(4):482-494

210. Zuo W et al (2014) Mitochondria autophagy is induced after hypoxic/ischemic stress in a Drp1 dependent manner: the role of inhibition of Drp1 in ischemic brain damage. Neuropharmacology $86: 103-115$

211. Zhang X et al (2013) Cerebral ischemia-reperfusion-induced autophagy protects against neuronal injury by mitochondrial clearance. Autophagy 9(9):1321-1333

212. Li Q et al (2014) Rapamycin attenuates mitochondrial dysfunction via activation of mitophagy in experimental ischemic stroke. Biochem Biophys Res Commun 444(2):182-188

213. Shi RY et al (2014) BNIP3 interacting with LC3 triggers excessive mitophagy in delayed neuronal death in stroke. CNS Neurosci Ther 20(12):1045-1055

214. Racay P et al (2009) Ischemia-reperfusion induces inhibition of mitochondrial protein synthesis and cytochrome c oxidase activity in rat hippocampus. Physiol Res 58(1):127-138

215. DeGracia DJ et al (2002) Molecular pathways of protein synthesis inhibition during brain reperfusion: implications for neuronal survival or death. J Cereb Blood Flow Metab 22(2):127-141

216. Sun N et al (2015) Measuring in vivo mitophagy. Mol Cell 60(4): 685-696 